\title{
Journal of Personality and Social Psychology
}

\section{Feelings Change: Accounting for Individual Differences in the}

Temporal Dynamics of Affect

Peter Kuppens, Zita Oravecz, and Francis Tuerlinckx

Online First Publication, September 20, 2010. doi: 10.1037/a0020962

CITATION

Kuppens, P., Oravecz, Z., \& Tuerlinckx, F. (2010, September 20). Feelings Change: Accounting for Individual Differences in the Temporal Dynamics of Affect. Journal of Personality and Social Psychology. Advance online publication. doi: 10.1037/a0020962 


\title{
Feelings Change: Accounting for Individual Differences in the Temporal Dynamics of Affect
}

\author{
Peter Kuppens \\ University of Leuven and University of Melbourne
}

\author{
Zita Oravecz and Francis Tuerlinckx \\ University of Leuven
}

\begin{abstract}
People display a remarkable variability in the patterns and trajectories with which their feelings change over time. In this article, we present a theoretical account for the dynamics of affect (DynAffect) that identifies the major processes underlying individual differences in the temporal dynamics of affective experiences. It is hypothesized that individuals are characterized by an affective home base, a baseline attractor state around which affect fluctuates. These fluctuations vary as the result of internal or external processes to which an individual is more or less sensitive and are regulated and tied back to the home base by the attractor strength. Individual differences in these 3 processes-affective home base, variability, and attractor strength—are proposed to underlie individual differences in affect dynamics. The DynAffect account is empirically evaluated by means of a diffusion modeling approach in 2 extensive experience-sampling studies on people's core affective experiences. The findings show that the model is capable of adequately capturing the observed dynamics in core affect across both large (Study 1) and shorter time scales (Study 2) and illuminate how the key processes are related to personality and emotion dispositions. Implications for the understanding of affect dynamics and affective dysfunctioning in psychopathology are also discussed.
\end{abstract}

Keywords: emotion, affect, dynamics, individual differences, attractor

A fundamental characteristic of emotions and affective experiences is that they change over time. Human lives are characterized by affective ups and downs, changes and fluctuations following the ebb and flow of daily life. In fact, the very reason why people have affective experiences in the first place is thought to lie in their dynamical nature. Affective changes inform people about important events that present a threat or opportunity to their well-being and allow them to respond to these changes with appropriate actions (Frijda, 2007; Larsen, 2000; Scherer, 2009). In short, people's affective lives only have meaning because they change.

Despite its central role, understanding the nature and processes underlying the temporal dynamics of affect and emotion remains one of the most important challenges in the study of emotion (Davidson, 2003; Lewis, 2005; Scherer, 2000b). One central insight that is emerging from research addressing this challenge is that how people's emotions and affective experiences change across time can be very different from one person to the next

Peter Kuppens, Department of Psychology, University of Leuven, Leuven, Belgium, and Department of Psychological Sciences, University of Melbourne, Melbourne, Victoria, Australia; Zita Oravecz and Francis Tuerlinckx, Department of Psychology, University of Leuven, Leuven, Belgium.

Peter Kuppens is a postdoctoral research fellow with the Fund for Scientific Research-Flanders (FWO). This research was supported by KULeuven Research Council Grant GOA/05/04. We are grateful to Andrew Gelman, Yoshi Kashima, Peter Koval, Stephen Loughnan, Batja Mesquita, Joachim Vandekerckhove, and Iven Van Mechelen for their helpful comments on the research reported in this article.

Correspondence concerning this article should be addressed to Peter Kuppens, Department of Psychology, University of Leuven, Tiensestraat 102, 3000 Leuven, Belgium. E-mail: peter.kuppens@psy.kuleuven.be
(Kuppens, Stouten, \& Mesquita, 2009). As noted by Davidson (1998), one of the most striking features of human emotions is indeed the broad variability across individuals in the patterns of changes that characterize their emotions. In the present article, we offer a theoretical account for understanding individual differences in the patterns and regularities characterizing affect dynamics. Building on central insights that have emerged from research on affect dynamics, we propose a theoretical model (labeled DynAffect) that identifies the basic processes underlying the changes and fluctuations in everyday affective experiences and that ties individual differences in these processes to more general personality and emotion dispositions.

\section{Individual Differences in the Dynamics of Affect}

The study of affect or emotion dynamics entails investigating the patterns and underlying processes that describe people's fluctuations and changes in emotion and its components across time. Until recently, most emotion research has focused on emotion or affect as a state and on identifying its antecedents and consequences (Kuppens et al., 2009; Scherer, 2000b). Yet emotion and affect are inherently dynamic in nature. Emotional and affective experiences are seen as the tools by which important internal and external changes are monitored and brought into consciousness (Carver \& Sheier, 1990; Scherer, 2009). Affective experiences may even become salient to consciousness only when they are subject to change, alerting people of any event that is important to their well-being (Russell, 2003). More and more research has therefore started to examine the patterns and regularities that drive the dynamics of affect. One direction has sought to identify the important ways people differ from each other in terms of affect dynamics, and several lines of findings are converging on what seem to be major sources of such differences. 
First, research has shown that people can be meaningfully characterized in terms of how they feel on average, demonstrating individual differences in average levels of positive and negative affectivity (e.g., Watson \& Tellegen, 1985), of pleasure and arousal (e.g., Kuppens, Van Mechelen, Nezlek, Dossche, \& Timmermans, 2007), as well as of more differentiated affective states (e.g., Diener, Smith, \& Fujita, 1995; Spielberger \& Sydeman, 1994). Moreover, such differences have proved to be associated with a broad range of other variables, such as personality (Rusting \& Larsen, 1995), mental health (Kuppens et al., 2007; Larsen \& Diener, 1985), and physical health (Cohen \& Pressman, 2006; Watson, 2000). From a dynamical perspective, such stable affect dispositions should be taken not as evidence that people are in a constant unchanging affective state but rather as an indication that people can be meaningfully characterized in terms of how they feel on average (Chow, Ram, Boker, Fujita, \& Clore, 2005; Larsen, 2000; Lykken \& Tellegen, 1996).

Second, it has become clear that people consistently differ in terms of how much their affective experiences vary across time. A growing body of research has established that individuals show stable differences in how strongly their emotions and affect fluctuate (Eaton \& Funder, 2001; Kuppens et al., 2007; Larsen, 1987; Penner, Shiffman, Paty, \& Fritzsche, 1994). Similar advances in other domains (e.g., regarding self-esteem: Kernis, Cornell, Sun, Berry, \& Harlow, 1993; interpersonal behavior: Moskowitz \& Zuroff, 2004; and also personality itself: Fleeson, 2001, 2004; Mischel \& Shoda, 1995) have led to the acceptance of the existence of stable individual differences in the temporal variability of affect and behavior. In the current context, Eid and Diener (1999) concluded that affect variability is sufficiently distinct from other traits to be considered a separate aspect of personality. Thus, an important element for understanding affect dynamics lies in the degree of temporal variability of affective experiences, with some people being more affectively stable than others.

Finally, human emotions and affective experiences do not come and go as they please (Gross, 2001) but are continuously regulated to meet hedonistic and instrumental motives (Tamir, 2009). Affect regulation refers to any process or strategy that is aimed at increasing, maintaining, or decreasing affective responses (Davidson, Jackson, \& Kalin, 2000; Gross, 2001), thereby redirecting the spontaneous flow of affective experiences (Koole, 2009). It is assumed that affect regulation is as ubiquitous as affective experience itself, in the sense that almost none of people's affective experiences are left untouched by regulation efforts. In fact, having an emotion means regulating the emotion to some extent in some way (Frijda, 2007; Scherer, 2000a). From the definition of affect regulation, it is obvious that it heavily affects the time course and dynamic patterns of affective experiences. Affective experiences can be strongly or mildly up-regulated or down-regulated, and this can impact many of the central temporal characteristics of emotion and affect, such as their duration (e.g., Verduyn, Delvaux, Van Coillie, Tuerlinckx, \& Van Mechelen, 2009), their intensity profile across time (e.g., Verduyn, Van Mechelen, Tuerlinckx, Meers, \& Van Coillie, 2009), their inertia (resistance to change; Kuppens, Allen, \& Sheeber, 2010), and in the end, perhaps most crucially, their return to baseline level (Chow et al., 2005; Davidson, 1998; Gross, 2002; Hemenover, 2003; Hemenover, Augustine, Shulman, Tran, \& Barlett, 2008; Koole, 2009; Larsen, 2000). Affect regulation is also considered to be subject to sizeable individual dif- ferences (Davidson, Jackson, \& Kalin, 2000; Gross \& John, 2003; Larsen, 2000), and such differences have been related to important outcomes, such as violence (Davidson, Putnam, \& Larson, 2000), depression (Davidson, Pizzagalli, Nitschke, \& Putnam, 2002), interpersonal relationships (Butler et al., 2003), and mental (Gross \& Muñoz, 1995) and physical well-being (Sapolsky, 2007). In sum, people are motivated to regulate their affective experiences, and this profoundly influences how affect unfolds over time.

\section{DynAffect: A Dynamic Process Account of Affective Change}

Despite the fact that previous research has been successful in identifying several of the fundamental ways people differ from one another in the dynamics of their affective experiences, the findings remain scattered, and an overarching theoretical account of affect dynamics is lacking. The aim of the present article is to integrate the previous findings into a unifying account that captures the key sources of individual differences in affect dynamics and to subject it to empirical study.

The DynAffect account we propose aims to explain individual differences in the dynamics of the two most fundamental properties of affect, namely, valence and arousal (Barrett \& Russell, 1999; Russell, 2003). As properties of people's subjective feelings, valence (ranging from feeling pleasant to feeling unpleasant) and arousal (ranging from feeling passive to feeling active) combine to form what has been labeled core affect, an integral state that is consciously accessible as a simple, nonreflective feeling (Barrett \& Bliss-Moreau, 2009; Russell, 2003). A person always has core affect, and at each point in time, a person's emotional state can be described in terms of how pleasant or unpleasant and how activated the person is feeling. Across time, a person's core affect describes a core affect trajectory (Kuppens et al., 2007), reflecting the typical pattern of affective changes and fluctuations that characterize an individual. People can show sizeable differences in their core affect trajectories. Figure 1 depicts core affect trajectories of two hypothetical individuals. Whereas Person 1 shows a relatively stable trajectory, the core affect changes of Person 2 are larger and more unstable. The current article aims to explain such differences.

The DynAffect account of core affect dynamics builds on the existing body of research on affect dynamics and is rooted in recent advances in theorizing about the dynamics of complex phenomena, in particular dynamical systems theory (e.g., Boker \& Wenger, 2007; Guastello, Koopmans, \& Pincus, 2009; Lewis, 2005; Thagard \& Nerb, 2002; Vallacher, Read, \& Nowak, 2002; van Montort, Oud, \& Satorra, 2007; Witherington \& Crichton, 2007). Dynamical systems theory has been developed in the natural sciences to account for often complex dynamical phenomena (see Schroeder, 1991; Strogatz, 1994), and many of the proposed models have also spurred interest in psychology and often found applications to account for the observed complexity in psychological data.

Starting from the assumption that core affect can be approached as resulting from a complex, open system, the DynAffect account proposes that the affective system of each individual is characterized by an affective home base, a set point that reflects the baseline functioning of the system. In dynamical systems terms, this home base represents the (fixed-point) attractor of the system (for more 

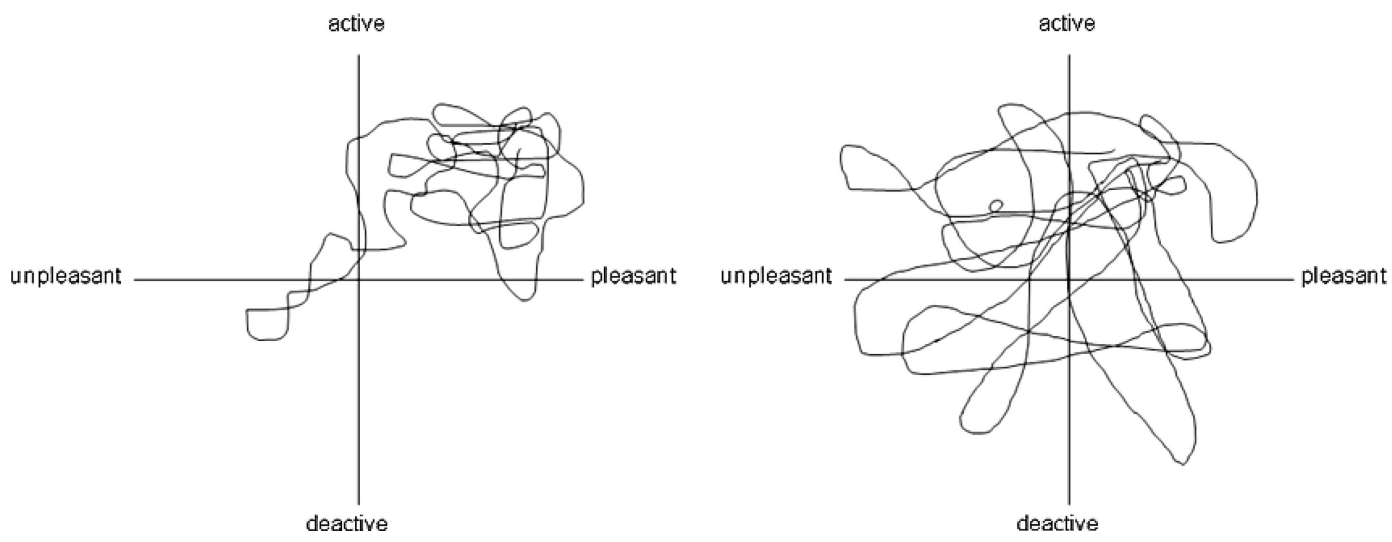

Figure 1. Core affect trajectories of two hypothetical individuals.

background, see, e.g., Guastello \& Liebovitch, 2009; Hoeksma, Oosterlaan, Schipper, \& Koot, 2007; Strogatz, 1994; Vallacher \& Nowak, 2009; Vallacher et al., 2002) and can be considered to reflect the affective comfort zone of an individual, signaling that everything is normal. Yet the open affect system is embedded in a larger context and is therefore subject to stochastic variability resulting from the many internal and external events that influence core affect. Such influences produce small or larger shifts in feeling and indicate that events are impinging on the person's core affect. The function of such changes to the system is to alert and motivate the person to respond to or cope with the events that are causing these changes (Frijda, 1986; Nesse \& Ellsworth, 2009; Russell, 2003; Scherer, 2009). However, the attractor keeps the system in balance by pulling core affect back to its home base, creating an emergent coherence around the attractor. The attractor strength reflects the regulatory processes that are installed to keep a person's core affect in check. We hypothesize that relatively enduring individual differences in these three key processesaffective home base, variability, and attractor strength — are largely responsible for producing the myriad ways people can display changes and fluctuations in their core affect throughout daily life. Figure 2 gives a graphical depiction of the three DynAffect processes, which we now explain in more detail.

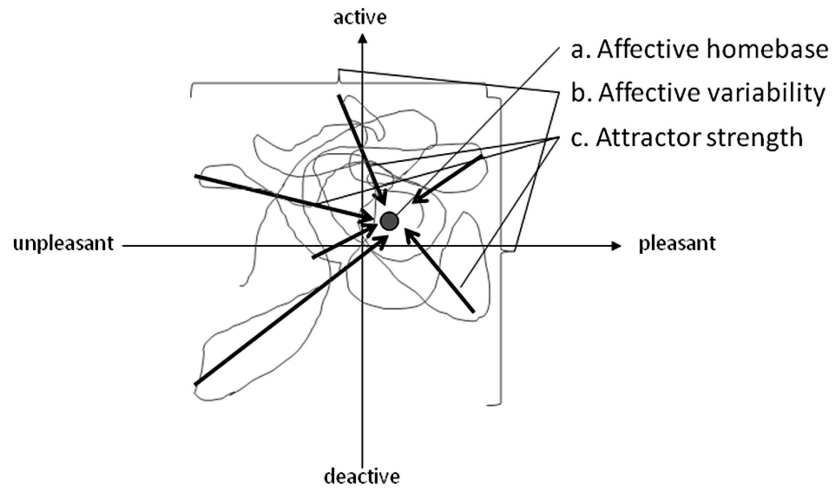

Figure 2. Graphical representation of the DynAffect processes that drive affective change: (a) affective home base, (b) affective variability, and (c) attractor strength.
The DynAffect account starts with the idea that people are characterized by an affective home base, a particular combination of valence and arousal values that reflects the typical affective state of the individual. The idea of an affective home base draws on theories that assume the existence of an affective set point or attractor state around which changes fluctuate (e.g., Carver \& Sheier, 1990; Headey \& Wearing, 1989; Hoeksma et al., 2007; Larsen, 2000). The basic idea is that people's affective changes revolve around a central focal point that serves as the affect system's baseline level, reflecting the default level of operation. ${ }^{1}$ Theoretically, the function of an attractor home base lies in acting as a reference point to which changes are compared, producing vital knowledge about the relation between the individual and his or her environment. Indeed, movements away from the baseline level signal deviations from a person's affective comfort zone, often therefore entering consciousness and causing changes in subjectively experienced affect (Russell, 2003). According to some, the default affective state of an organism is characterized by slight positive valence and arousal levels, motivating the organism to approach novel stimuli and environments and enabling it to learn and explore (e.g., Cacioppo \& Gardner, 1999). Yet both genetic (e.g., Lykken \& Tellegen, 1996) and environmental (e.g., Diener \& Diener, 1996; Lucas, 2007) factors contribute to the creation of sizeable individual differences in affective baseline levels (Kuppens, Tuerlinckx, Russell, \& Barrett, 2010). In other words, a person's affective home base can be more or less pleasant and more or less active on average. It is the existence of these individual differences in affective home base that is observed in research on stable individual differences in average affect.

Second, DynAffect posits a stochastic process that reflects the changes and fluctuations that people's pleasure and arousal levels undergo across time and that accounts for the observed individual differences in core affect variability. Core affect is continuously influenced by external and internal events, lending it its dynamic

\footnotetext{
${ }^{1}$ By proposing the affective home base as a relatively enduring set point, we do not imply that individual differences in average affect levels cannot change. As has become clear, how one feels on average can change over the course of one's life as a function of, for instance, major life events (e.g., Lucas, 2007), as well as through effortful interventions and practice (Lyubomirsky, Sheldon, \& Schkade, 2005).
} 
nature (Russell, 2003, 2009). The factors that cause these smaller and larger affective shifts are many and intertwined (Barrett, Mesquita, Ochsner, \& Gross, 2007), starting with the subjective appraisal of ongoing events (Scherer, Dan, \& Flykt, 2006) and biological factors such as biobehavioral feedback (Niedenthal, 2007), hormone levels (Susman, Dorn, \& Chrousos, 1991), and substance use (Nesse $\&$ Berridge, 1997), as well as more mundane factors such as time of the day (e.g., Kahneman, Krueger, Schkade, Schwarz, \& Stone, 2004), the weather (e.g., Denissen, Butalid, Penke, \& van Aken, 2008), physical activity (e.g., Ekkekakis, 2009), music (e.g., Grewe, Nagel, Kopiez, \& Altenmüller, 2007), and so on. The result of the complex combination of such influences is the emergence of a certain level of variability in people's affective experiences across time. From a mere individual-differences perspective, the task here is not to identify and incorporate all these factors but to take into account their combined impact on the changes in a person's affective experiences. Due to dispositional differences between individuals in differentially selecting (Ickes, Snyder, \& Garcia, 1997) and reacting to (e.g., Larsen \& Ketelaar, 1991) the different factors that influence core affect, stable individual differences in affect variability emerge. In core affect terms, this means that people can show more or less variability with respect to how pleasant or unpleasant they are feeling (valence variability) and with respect to how active or passive they are feeling (arousal variability).

Finally, this stochastic variability is tied to the affective home base with a certain degree of attractor strength. Theories of emotion or affect dynamics assume that regulatory processes are activated to regulate affective states that deviate from the set point or baseline level (e.g., Chow et al., 2005; Larsen, 2000). Theoretically, the function of the attraction to the home base is to prevent the system from reaching extreme values that impair normal functioning of the system. When events make a person's feeling state shift away from its affective home base, regulatory processes aimed at redirecting core affect back toward the home base are called into action. Without such regulatory process, affect would linger endlessly (and to dangerous extremes), with no motivation to return to the baseline level needed for normal functioning and as a comparison state for novel input. Such regulatory processes represent affect regulation in its most basic form and have been documented under various forms, such as affect repair (Hemenover, 2003; Hemenover et al., 2008), the principle of a thermostat (e.g., Chow et al., 2005), feedback loops (Carver \& Sheier, 1990), and down-regulatory processes (Gross, 1998; Koole, 2009), and can be seen as the conceptual opposite of emotional inertia (Kuppens et al., 2010).

In sum, the DynAffect account proposes that the following fundamental processes drive individual differences in core affect dynamics: affective home base, affective variability, and attractor strength, with each operating separately on the valence and arousal properties of core affect. Our account states that individual differences in both the coherence and variability of affect dynamics emerge as a result of the complex interactive effects of the multiple factors implicated in affect reactivity and regulation and that such differences can be grasped by individual differences in these three key concepts. We do not assume that DynAffect covers all possible processes that come into play for explaining individual differences in how affect unfolds across time. Yet we propose that it allows the capture of the most fundamental ways individuals differ in terms of how their core affect fluctuates, enabling an account of a wide variety of observed core affect patterns and trajectories.

\section{Relation Between DynAffect Processes and Existing Dispositional Characteristics}

On the basis of previous research, we expect the DynAffect processes to be associated with (or to reflect) several individual differences in the domains of personality, adjustment, and emotion regulation, and empirical evidence for such associations would provide support for the validity of the account. From the personality domain, we expect the valence dimension of affective home base to be negatively related to neuroticism and positively related to extraversion (e.g., Larsen \& Ketelaar, 1991; Rusting \& Larsen, 1995). Trait negative and positive affect (Watson, Clark, \& Tellegen, 1988) are expected to display similar relationships with valence home base. Moreover, as neuroticism is seen as a key predictor or indicator of affective instability (e.g., Eid \& Diener, 1999; Hepburn \& Eysenck, 1989; Kuppens et al., 2007), it is expected to be positively related to affective variability.

From the domain of psychological adjustment and well-being, research has shown that psychological adjustment entails experiencing relatively more positive and fewer negative emotions (Diener, Oishi, \& Lucas, 2003; Fredrickson \& Losada, 2005) and a more stable emotional life (e.g., Jones, 2006; Kuppens et al., 2007). Consequently, we hypothesize that the valence of affective home base will be positively, and affect variability be negatively, related to indicators of psychological well-being such as selfesteem and satisfaction with life. Moreover, self-esteem at the state level is seen as an important determinant of how people feel from moment to moment-how people value themselves seems to run parallel with how they feel emotionally (e.g., Kernis, 2003). As a result, an unstable self-esteem should therefore be related to affective variability (e.g., Oosterwegel, Field, Hart, \& Anderson, 2001).

Finally, moving to theoretically more novel predictions, we expect that attractor strength is at least in part a function of emotion regulation and that the habitual use of particular regulation strategies (be it willful or not) is related to individual differences in attractor strength. Emotion regulation by reappraisal is meant to change cognitions about the emotion-eliciting stimulus so as to decrease the emotional impact of emotion-eliciting events (e.g., Gross, 1998). Consequently, we expect habitual reappraisal to be related to increased attractor strength. The regulation strategy of suppression is not expected to impact experiential changes as it targets the behavioral expression of emotion (Gross, 2001). Finally, rumination refers to repetitively focusing on the emotioneliciting event and has been associated with prolonged negative emotionality (e.g., Rusting \& Nolen-Hoeksema, 1998). Rumination may therefore impede returning to baseline affect and is therefore expected to be associated with decreased attractor strength.

\section{Empirically Testing the DynAffect Account}

In the previous sections, we have given a verbal account of the most important principles of the DynAffect account. To make it amenable to empirical research and test predictions derived from it, the ideas and principles put forward in the previous sections 
need to be translated into a statistical model that allows the testing and making of inferences about the hypothesized processes (see also Hoeksma et al., 2007; for a general argument for a modeling approach, see Rodgers, 2010).

The mathematical tools for modeling dynamics with stochastic variability are stochastic differential equations (see, e.g., Gardiner, 2004), also known as diffusion models. ${ }^{2}$ For the present purpose, we formulated a hierarchical (i.e., random effects) diffusion model that reflects a direct mathematical conceptualization of the principles and assumptions of our theoretical DynAffect account (Oravecz \& Tuerlinckx, 2008; Oravecz, Tuerlinckx, \& Vandekerckhove, 2009, in press).

The core of the model can be described in terms of two equations. For simplicity, we discuss these for the case of modeling one dimension (instead of two), although the eventual analyses are based on a model for two dimensions (valence and arousal). The generalization to two dimensions follows logically from the onedimensional model (see Oravecz et al., in press). If $Y_{p}(t)$ is the observed position for person $p$ at time $t$ on an affective dimension, the diffusion model can be described in terms of the following two equations (i.e., the measurement equation and the transition equation):

$$
\begin{array}{rlr}
Y_{p}(t) & =\theta_{p}(t)+\varepsilon_{p}(t) . & \text { (measurement equation) } \\
\mathrm{d} \theta_{p}(t) / \mathrm{d} t & =\beta_{p} \times\left(\mu_{p}-\theta_{p}[t]\right)+\xi_{\mathrm{p}}(t) . & \text { (transition equation) }
\end{array}
$$

The measurement equation shows a resemblance to many statistical models in the behavioral sciences and states that the observed value for person $p$ at time $t$ (i.e., $Y_{p}[t]$ ) is a measurement errorcorrupted version of a latent true score for that person $p$ at time $t$ (i.e., $\left.\theta_{p}[t]\right)$. The transition equation expresses the rule for the change in the latent true score $\theta_{p}(t)$ across time and harbors the parameters reflecting the DynAffect processes of home base, variability, and attractor strength. In particular, the instantaneous change in $\theta_{p}(t)$ (i.e., the first derivative with respect to time, $\left.\mathrm{d} \theta_{p}[t] / \mathrm{d} t\right)$ is a function of the signed distance between the home base and the current position $\left(\mu_{p}-\theta_{p}[t]\right)$ multiplied by the value for the attractor strength for that person (i.e., $\beta_{p}$ ). In addition, the random noise parameter $\xi_{\mathrm{p}}(t)$ represents the influences of all factors producing changes in core affect and determines, together with the attractor strength, the variability in the dynamical process. All parameters in the two equations are allowed to differ over persons (see the index $p$ ).

The model's parameters directly represent the key DynAffect processes described above (home base, variability, and attractor strength) and therefore allow a direct test of our hypotheses about the processes underlying core affect dynamics. By applying this model to affect dynamical data and evaluating how well it is capable of describing these data (in terms of relative and absolute fit), we can assess to what extent the DynAffect model processes are capable of accounting for observed patterns of affective change (Rodgers, 2010). Moreover, the model allows the study of individual differences in the hypothesized processes because the parameters of the diffusion model are allowed to take on different values across individuals by assuming that they are drawn from a population distribution, as in traditional multilevel models (e.g., Snijders \& Bosker, 1999). Also, the model assumes time to be continuous (as opposed to discrete, as in traditional time-series analysis). This is important because even when affect may only be measured at discrete time points, it can be considered to unfold continuously between measurement moments.

The DynAffect account and its predictions were empirically evaluated in two studies by applying the proposed diffusion model to experience-sampling data on people's core affect throughout their daily lives. Experience-sampling methods (e.g., Csikszentmihalyi \& Larsen, 1987) allow one to collect information on people's affective states throughout the course of their normal, daily life, thereby capturing life as it is lived (Bolger, Davis, \& Rafaeli, 2003). The resulting data are highly ecologically valid as data collection occurs within the real-life circumstances of the participant, not in artificial contexts (Barrett \& Barrett, 2001), and are less affected by recall biases than retrospective self-reports (e.g., Stone et al., 1998). In Study 1, participants recorded their core affect for 10 times a day over a period of 2 weeks, yielding ecologically valid data on the dynamics of affect over the course of hours, days, and weeks. In Study 2, participants recorded their core affect 50 times a day over 4 days, yielding information on core affect transitions over the course of minutes.

\section{Study 1}

\section{Method}

Participants. 80 university students from the University of Leuven in Belgium took part in the study. One participant quit the study after one day of sampling, resulting in a final sample of 79 participants (50 female, mean age $=24$ years). Participants were paid $40 €$ for their participation (approximately \$53 U.S.).

\section{Materials.}

Repeated assessment of core affect. Core affect at each sampling moment was assessed using a modified version of the Affect Grid, a single-item measure designed to simultaneously assess subjectively felt valence and arousal (Russell, Weiss, \& Mendelsohn, 1989). The modified version consists of a $99 \times 99$ (instead of $9 \times 9$ ) two-dimensional grid with a neutral middle row and middle column. Unpleasant-pleasant feelings form the horizontal dimension, arousal-sleepiness the vertical. End- and midpoints are marked with affective labels to facilitate reporting. Participants were instructed to mark the position on the Affect Grid that best corresponded to how they felt at each sampling moment (signaled by a beep). The one-item Affect Grid is ideally suited for repeatedly assessing core affect in the context of experience sampling as it does not overload the participant and enables a quick response.

Repeated assessment of self-esteem. At each sampling moment, participants were also asked to answer a number of other

\footnotetext{
${ }^{2}$ The first diffusion model was derived by Einstein (1905) to explain the erratic movement of a small particle (e.g., small parts of a pollen grain) immersed in a liquid as a result of incessant impacts with the liquid's molecules (also called Brownian motion after the Scottish botanist who first observed it; Brown, 1828). Later, Uhlenbeck and Ornstein (1930) expanded diffusion models to incorporate an equilibrium state to which the Brownian motion is tied, which would later be conceptualized as an attractor. Nowadays, the mathematical properties of this model are well understood (see, e.g., Cox \& Miller, 1965), and it (or related versions) is applied in such diverse areas as physics (Mazzo, 2002), finance (BarndorffNielsen \& Shepard, 2001), sociology (Oud, 2007), animal ecology (Blackwell, 1997), and psychology (Oravecz, Tuerlinckx, \& Vandekerckhove, in press).
} 
questions. Of interest to the current study, one item assessed momentary self-esteem ("How much self-confidence do you have at this moment?") and was rated by using a continuous slider scale (ranging from $0=$ none to $100=$ very much). This was used to obtain a measure of self-esteem variability by taking the standard deviation across sampling moments per participant.

\section{Dispositional questionnaires.}

Neuroticism and extraversion. Neuroticism and extraversion were measured with the 12-item (rated on a 5-point scale ranging from $1=$ completely disagree to $5=$ completely agree) scales from the Dutch version of the NEO Five-Factor Inventory (Hoekstra, Ormel, \& De Fruyt, 1996). Cronbach's alphas equaled .88 and 80 , respectively.

Positive and negative affect. Positive and negative affect were measured using the Positive and Negative Affect Schedule (Watson et al., 1988), which consists of 20 affective adjectives (10 positive, 10 negative) to be rated on a 5-point scale (ranging from $1=$ not at all to $5=$ very strong) in terms of how one normally feels. Cronbach's alphas equaled .78 and .85 , respectively.

Self-esteem. The Rosenberg (1989) self-esteem scale consists of five positively and five negatively formulated items that measure a person's stable sense of self-esteem. Each item has to be rated on a 4-point scale $(1=$ does not describe me at all to $4=$ describes me very well). Cronbach's alpha equaled .86 .

Satisfaction with life. Life satisfaction was assessed with the Satisfaction With Life Scale (Diener, Emmons, Larsen, \& Griffin, 1985; Pavot \& Diener, 1993). The scale consists of five items that are rated on a 7-point scale (ranging from $1=$ strongly disagree to 7 = strongly agree). Cronbach's alpha equaled .87 .

Reappraisal and suppression. The Emotion Regulation Scale (Gross \& John, 2003) consists of 10 items that tap the habitual use of reappraisal and suppression (rated on a 7-point scale ranging from 1 = completely disagree to 7 = completely agree). Cronbach's alphas equaled .68 and .78 , respectively.

Rumination. The rumination scale from Trapnell and Campbell (1999) measures the tendency for brooding and recurrent thinking over one's problems or negative emotions and consists of 12 items that are rated on a 5-point scale (ranging from $1=$ totally disagree to $5=$ totally agree). Cronbach's alpha equaled .91 .

Procedure. In a first session, each participant received a Tungsten E2 palmtop computer along with instructions for its use in general as well as for responding to the questions at each beep, including elaborate instructions for the Affect Grid (see Russell et al., 1989). Each palmtop was programmed to beep 10 times a day for 14 consecutive days during the participant's waking hours. The beeps were programmed according to a stratified random interval scheme: Participant's waking hours were divided into 10 equal intervals, and one beep was programmed randomly in each interval. At each beep, the palmtop presented a number of questions in randomized order, including the Affect Grid and self-esteem item. In the first session, participants also completed half of the dispositional questionnaires (randomized between participants). For the next 2 weeks, participants carried the palmtop during their normal daily activities and responded to the questions when signaled. Compliance was good: Overall, participants responded to $82 \%$ of the programmed beeps. After 2 weeks, participants attended a second session in which they completed the remaining questionnaires and were paid for participation.
Analyses. The diffusion model with person-specific values for all its leading parameters cannot be estimated in mainstream scientific software packages like HLM or SAS (see Oravecz \& Tuerlinckx, in press, for an extensive comparison with related models). Instead, statistical inference is carried out within a Bayesian statistical framework using a custom-made program in MATLAB. ${ }^{3}$ The use of Bayesian statistics is becoming increasingly widespread in psychological research, both as a principled way of statistical reasoning (see, e.g., Lee, 2008; Rouder, Speckman, Sun, Morey, \& Iverson, 2009) and as a tool to perform statistical inference in complex models (see, e.g., Rouder, Tuerlinckx, Speckman, Lu, \& Gomez, 2008). A detailed description of the model and of the statistical inference of the model is given in Oravecz and Tuerlinckx (2008) and Oravecz et al. (2009, in press). ${ }^{4}$ Missing data are handled straightforwardly due to the continuous time nature of the model.

\section{Results}

Model fit. Traditional fit indices like $R^{2}$ are not available in the diffusion model framework for several reasons. ${ }^{5}$ We therefore present a set of other indices to evaluate model fit. The chosen strategy for testing model fit can be subdivided into three subcategories. First, we followed a model-selection strategy to assess relative fit by investigating the goodness of fit of the DynAffect diffusion model in comparison to a set of alternative models (in which one or more aspects of the DynAffect model are absent).

\footnotetext{
${ }^{3}$ If we denote observed data in a generic way as $Y$ and a model's parameters as $\theta$, then in Bayesian statistics, inferences are based on the posterior distribution, which is defined as follows: $p(\theta \mid Y)=p(Y \mid \theta) \times$ $p(\theta) / p(Y)$, where $p(\theta \mid Y)$ is the posterior distribution, $p(Y \mid \theta)$ is the likelihood (as in frequentist or classical statistics), $p(\theta)$ is the prior distribution on the parameters, and $p(Y)$ is a normalization term, such that $p(Y \mid \theta) \times p(\theta)$ is normalized and thus integrates to one over the parameters. In fact, the above equation is simply Bayes's theorem applied to the model's parameters treating the observed data as given. All inferences regarding the model's parameters are based on the posterior distribution. In complex models (such as the diffusion model in this article), the Bayesian framework is extremely helpful because one may summarize the posterior distribution by drawing samples from it and applying summary measures to these samples (e.g., the mean, variance, etc.). Often, the posterior is not of a known form (e.g., the normal or the $t$ distribution) and is only known up to a constant of proportionality (i.e., the normalizing factor $p[Y]$ is not easy to compute). However, we made use of computational methods not hampered by these aforementioned obstacles (so-called Markov chain Monte Carlo methods; see Gelman, Carlin, Stern, \& Rubin, 2004; Tanner, 1996) to draw samples from the posterior.

${ }^{4}$ The studies that introduced the DynAffect diffusion model are available upon request from us as well as on the website http://ppw.kuleuven.be/ okp/home/ (for published studies). In the future, easily accessible software packages will be published on this website to allow researchers to apply the DynAffect diffusion model to their own data. Meanwhile, interested researchers are welcome to contact us with any queries.

${ }^{5}$ First, we do not deal with nested models with varying numbers of covariates as is usually the case in, for example, linear regression modeling. Second, although $R^{2}$ measures for hierarchical linear models have been developed (see Gelman \& Hill, 2007; Snijders \& Bosker, 1999), this has not yet been pursued to the same extent as for nonhierarchical linear models. To the best of our knowledge, $R^{2}$ analogue measures for models as applied in the current article have not yet been constructed and validated.
} 
Second, we tested how well the selected model described the data by comparing critical aspects of observed data to the same aspects of data replicated under the DynAffect model. The idea behind this technique is that if the DynAffect diffusion model is indeed (approximately) the model generating the data, we should find that specific patterns in the observed data closely resemble those from data sets simulated from the model. Finally, we explored how well the model could predict future data.

Model selection: Relative goodness of fit. Because the Bayesian framework precludes the use of traditional model-selection tools like the Akaike information criterion (AIC) or Bayesian information criterion (BIC), we made use of the deviance information criterion (DIC) developed for a Bayesian context (Spiegelhalter, Best, Carlin, \& van der Linde, 2002). Like the AIC and BIC, the DIC trades off two important features of the model: fit (measured by a deviance statistic) and complexity (based on the number of the parameters). The model with the smaller DIC provides the best fit taking into account model complexity and is selected. The models that were compared to each other were the DynAffect diffusion model in which all parameters are person specific (reflecting the complete dynamical account outlined above; this is also the most complex model) and a number of simpler models in which specific parameters are kept constant across individuals or in which they are restricted to a particular value. Alternative Models 1-3 assume no individual differences in attractor strength, affective variability, or both, respectively (the home-base parameter always varied randomly because preliminary analyses evidenced substantial individual differences in average core affect values; including models with a fixed home base did not change conclusions). As the centralizing tendency is an essential model assumption, we tested that aspect by fixing it to 0 in a fourth alternative model. This model assumes the absence of attractor strength and conceptually corresponds to a model for simple random walk or Brownian motion (e.g., affect changes randomly without being attracted to a home base). Finally, in Alternative Model 5, the centralizing tendency is set to infinity, which comes down to a simple bivariate model that does not take time into account at all. ${ }^{6}$ Table 1 lists the DIC values of all estimated models. The lowest DIC is clearly observed for the DynAffect diffusion model, strongly indicating that all the parameters reflect essential processes for accounting for observed core affect dynamics. All following analyses are based on this model. Importantly, the models with the attractor strength set to 0 or infinity proved to be the worst, evidencing that the attractor element and time dependency are crucial for accounting for changes in core affect.

How well does the selected model fit the observed data? Comparing observed and replicated core affect trajectories. Plotting for each person the observations in a two-dimensional core affect space and connecting subsequent points with a line results in an observed person-specific core affect trajectory (Kuppens et al., 2007). On the basis of the fitted diffusion model, such trajectories can also be simulated or predicted from the model (keeping the same time differences as in the observed data). If the model fits the data, the replicated trajectories should closely resemble the observed trajectories in shape and dispersion. Figure 3 illustrates this notion graphically by displaying the observed trajectories of 10 participants (characterized by different levels of home base, variability, and attractor strength) along with two trajectories that were simulated under the DynAffect model with the parameters set to the estimated values for that individual. It is important to stress that the replicated trajectories cannot follow exactly the same path as the observed trajectory because the model is inherently stochastic. Notwithstanding, the graphs in Figure 3 illustrate that the spatial characteristics of the observed trajectories are preserved very well in the replicated ones.

Besides this purely visual assessment of the similarity between the observed and replicated trajectories, we also examined the overlap between the observed and simulated trajectories quantitatively by calculating the correlation between the frequencies that the observed data fall in a certain area of the core affect space and the average frequency that they fall in that same area across replicated data sets. Distinguishing between low, average, and high pleasantness and activation values yields nine basic areas that correspond to substantively meaningful different feeling states (e.g., neutral valence-low arousal, pleasant valence-low arousal, etc.). If we calculate the visit frequency in these areas for every person and correlate it with the corresponding simulated mean visit frequencies (across replicated data sets), we can see how well the model does in recovering how frequently each individual feels these different states. On the basis of 1,000 replications per person, the average correlation per person was $0.80(S D=0.20$; even when making use of a much more fine-grained $9 \times 9$ division of the core affect space, the average correlation was still 0.47 ). This shows that the DynAffect model provides a good estimation of how often people are in qualitatively different feeling states.

In addition to focusing on the full trajectories and visited areas, we also examined the recovery of a purely dynamical aspect of the data in terms of vector fields. After pooling the data from all participants, we divided the core affect space into a $9 \times 9$ grid and calculated a vector for each cell. The length of the vector is proportional to the average speed of escape from the cell (i.e., when a participant feels a certain way corresponding to the location of the cell, how fast does that feeling change?), and the direction indicates to which area it tends toward (i.e., when a participant feels a certain way, how will he or she tend to feel next?) and captures purely dynamical features of the trajectories. In Figure 4, the black lines correspond to the vectors calculated from the observed data. We can observe that at the average position in the grid (close to the middle where the population home base lies; for the exact value of the population home base, see Table 2), the average speed is very small (i.e., short vectors), but the farther we move from the average position, the higher the escape speed becomes. Most vectors point more or less directly to

\footnotetext{
${ }^{6}$ A model with infinite attractor strength simplifies to a simple bivariate model that assumes that people's individual core affect trajectories can be modeled in terms of their means, variances, and covariances only, in which all parameters are again person specific. It should be noted that in such a model, (a) the time dependency of the data is not taken into account at all (because the attractor strength is inversely related to the autocorrelation, so when the strength is infinite, the autocorrelation is zero) and (b) it is impossible to separate measurement error from intraindividual stochastics at the latent level. Because of the second property, it has to be noted that the model structure changes fundamentally (e.g., the previously presented measurement equation is dropped from the model), and as a consequence, the way the DIC is computed in this model differs from the DIC computation in the other models.
} 
Table 1

Relative Goodness-of-Fit DIC Measure for the DynAffect Diffusion Model and for Five Alternative Less Complex Models (Study 1)

\begin{tabular}{|c|c|c|c|c|}
\hline Model & Home base & Variability & Attractor strength & Estimated DIC \\
\hline DynAffect & random & random & random & $-3,604$ \\
\hline Alternative 1 & random & random & fixed & 1,966 \\
\hline Alternative 2 & random & fixed & random & 5,151 \\
\hline Alternative 3 & random & fixed & fixed & 15,872 \\
\hline Alternative 4 & random & random & 0 & 20,408 \\
\hline Alternative 5 & random & random & infinite & 40,082 \\
\hline
\end{tabular}

Note. Random indicates that the parameters vary across persons, and fixed indicates that all persons posses the same value. The best fitting model is displayed in bold. DIC = deviance information criterion.

the central location, and with increasing distance from the central point, the vector length increases. Next, the grey lines in Figure 4 are escape velocity vectors based on the replicated data sets (20 in total). It can be seen that for the majority of the cells, the observed velocity vector (in black) falls within the range of velocity vectors predicted by the model. ${ }^{7}$

Predictions of future data points based on the selected model: Prediction of the final observation. As a final way of model checking, we assessed how well the model was able to predict the final observation for each person based on model parameters estimated from data that did not include that observation. In other words, if we knew a person's affective home base, variability, and attractor strength based on how they have been feeling until now, would we be able to predict how that person would feel next? On the basis of simulated data, the final observation was predicted 1,000 times. From these 1,000 replications, we specified a $95 \%$ prediction interval for both valence and arousal. For valence, $80 \%$ of the observed last points fell into this interval; for arousal, $91 \%$. Although this indicates that there is some undercoverage (i.e., if the model fits perfectly, we would expect to cover the final observation about $95 \%$ of the times), these results nevertheless demonstrate that our prediction uncertainty is well calibrated.

Descriptive statistics and correlations with trait measures. Table 2 summarizes descriptive statistics of the DynAffect parameters on the population level (i.e., the average estimates and their standard deviations) and their intercorrelations across participants. To aid comparison with previous studies, the observations were rescaled between 0.1 and 9.9 (comparable to the values obtained with the original $9 \times 9$ grid). In line with previous findings (Kuppens et al., 2007), it can be seen that the average home base is slightly pleasant and of medium arousal and that people display substantial amount of variability on the two dimensions, with variability in arousal being slightly larger than variability in valence (see also Kuppens et al., 2007, Study 1). The intercorrelations show small to moderate associations among the parameters across individuals. Of note are the positive correlation between valence and arousal variability (see also Kuppens et al., 2007) and the positive correlation between arousal variability and the valence of the home base (indicating that arousal variability is related to feeling more pleasant on average). The negative correlation between valence and arousal home base, however, is inconsistent with previous findings (Kuppens et al., 2010). It remains to be seen whether this association is found consistently using the current approach or whether it is specific to the current sample.
Finally, Table 3 reports the correlations between the personspecific estimates and the dispositional measures. Extraversion, positive affect, self-esteem, and life satisfaction were related to a more pleasant home base, whereas neuroticism and negative affect were associated with a more unpleasant home base. In turn, home base arousal level was positively associated with neuroticism and suppression and negatively associated with self-esteem. Valence variability was found to be negatively related to self-esteem and positively related to neuroticism, negative affect, and self-esteem variability. The latter was also positively associated with arousal variability. Finally, reappraisal and rumination were positively and negatively, respectively, related to arousal attractor strength.

\section{Discussion}

Study 1 provided converging evidence that the DynAffect model adequately captures the affect dynamics observed in participants' core affect throughout their daily lives. The results relating to model fit showed that the DynAffect model is the best fitting model compared with alternative models that do not assume individual differences in one of the key processes or do not involve the notion of an attractor. Furthermore, data simulated under the DynAffect model very well replicated characteristics of the observed data, and the model was capable of predicting how a participant would feel next based on knowledge of the participant's model parameters. In all, these results strongly suggest that the notions of home base, variability, and attractor strength put forward in the DynAffect account reflect key processes driving individual differences in the temporal dynamics of core affect.

Moreover, the correlations between the DynAffect parameters and the dispositional trait measures provided convergent validity about the nature of these processes. As expected, indicators of positive emotionality and well-being were associated with a more pleasant home base and vice versa. In turn, neuroticism and low self-esteem were related to a higher arousal home-base level, as was the emotion regulation strategy of suppression. The latter is an interesting novel finding because it provides a self-report analogue of experimental findings demonstrating that suppression leads to

\footnotetext{
${ }^{7}$ A few exceptions occur, mostly on the left side (low pleasantness), where there is somewhat more deviation between the observed and the replicated data. This is due to the fact that there are only a couple of observations with very low pleasantness values, and the observed data vectors could therefore be calculated on the basis of only a handful of data points.
} 


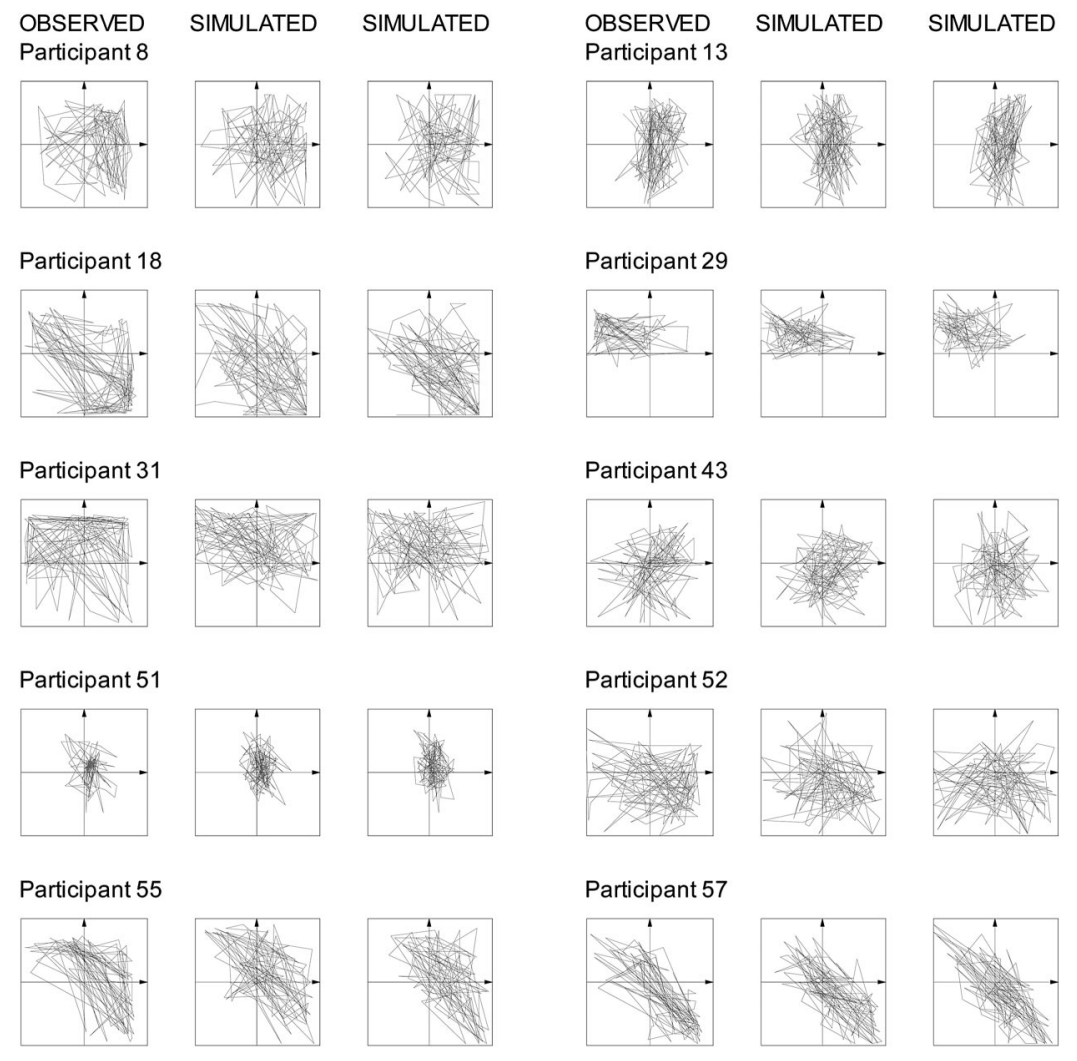

Figure 3. Observed core affect trajectories of 10 participants with two corresponding replicated trajectories based on data simulated under a DynAffect diffusion model implemented with the participants' estimated affective home base, variability, and attractor strength parameters (Study 1).

increased physiological arousal (Gross, 1998; Gross \& Levenson, 1997). Primarily, valence instability was related to negative emotionality and low well-being, whereas arousal variability was less related to such indicators of maladjustment. Both were related to self-esteem variability, however, once again illustrating that how one thinks about oneself and how one feels go hand in hand (Nezlek \& Plesko, 2001). Going beyond previous research, the results showed that habitual reappraisal is associated with a stronger attractor strength for arousal. In contrast, people who tend to ruminate are characterized by a decreased attractor strength for arousal. These results show that individual differences in attractor strength are related to the use of emotion regulation strategies and moreover suggest that these strategies may primarily impact the arousal property of affect.

\section{Study 2}

Both longer lasting affective fluctuations (Larsen, 2000; Murray, Allen, \& Trinder, 2002) and shorter lived emotional episodes (Koole, 2009) are characterized by chronic tendencies and temporal variability, as well as being subject to regulatory efforts. Our expectation is that the DynAffect principles of affective home base, variability, and attractor strength may therefore apply to both changes observed across hours and days and changes and fluctuations observed across minutes, even though it is likely that some of the underlying processes may not be of the same nature. The main purpose of this second study was therefore to replicate the applicability of the DynAffect model for affect dynamics on a much shorter time scale compared to Study 1 and to examine corresponding trait correlates. An experience-sampling study was set up in which participants' core affect was assessed 5 times more frequently than in Study 1, yielding information on core affect approximately every $17 \mathrm{~min}$ on average (compared to approximately every $84 \mathrm{~min}$ in Study 1 ).

\section{Method}

Participants. 60 university students from the University of Leuven in Belgium (40 female, mean age $=23$ years) were recruited through the university job service center and paid $50 €$ for participation (approximately $\$ 65$ U.S.).

\section{Materials.}

Repeated assessment of core affect. Core affect reports at each sampling moment were again recorded using a modified version of the Affect Grid identical to the one used in Study 1. Given the burden on participants caused by the intense sampling scheme, momentary self-esteem was not assessed.

Dispositional questionnaires. The same questionnaires as in Study 1 were used to assess dispositional neuroticism and extraversion (Hoekstra et al., 1996), positive and negative affect (Watson et al., 1988), self-esteem (Rosenberg, 1989), satisfaction with life (Diener et al., 1985), reappraisal and suppression (Gross 


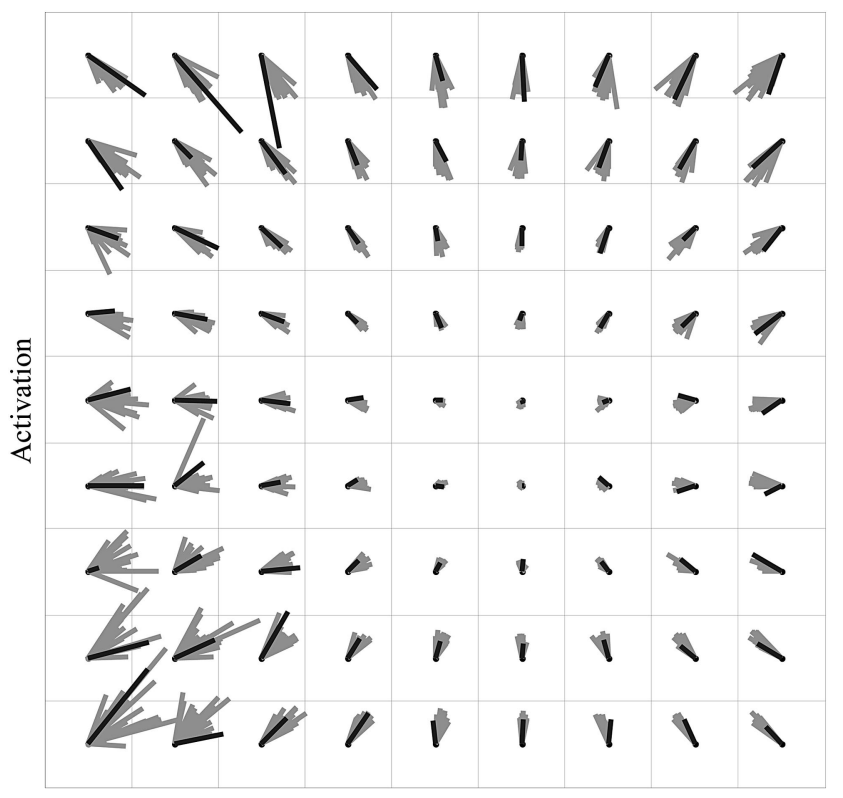

Pleasantness

Figure 4. Pooled vector field plot of the core affect space. The black lines reflect the observed vector (with the length reflecting the escape velocity and the direction the escape direction); the grey lines reflect vectors calculated on the basis of 20 simulated replications of the data under the DynAffect diffusion model (Study 1).

\& John, 2003), and rumination (Trapnell \& Campbell, 1999). Cronbach's alpha's for these scales equaled $.85, .85, .84, .83, .88$, $.81, .71, .78$, and .92 , respectively.

Procedure. In a first session, each participant received a Tungsten E2 palmtop computer along with instructions (see Study 1). Each palmtop was programmed to beep 50 times a day for 4 consecutive days during the participant's waking hours. As in Study 1, the beeps were programmed according to a stratified random interval scheme. The participants were prepared at length for the intensive sampling scheme, appealing to both intrinsic (the importance of compliance to ensure the quality and validity of the data) and extrinsic (the size of the monetary reward) motivation. Participants were explicitly told that the intensive data collection might become cumbersome at times but were motivated to respond to as many of the programmed beeps as possible. In the first session, participants completed half of the dispositional questionnaires (randomized between participants). After 5 days, participants attended a second session in which they completed the second half of the questionnaires and were paid for participation. Taking into account the intensive nature of the data collection and the intrusion this implied on participants' daily activities, compliance was very good: In total, participants responded to $87 \%$ of the programmed beeps.

\section{Results}

\section{Model fit.}

Model selection: Relative goodness of fit. DIC values were calculated for the same set of models as in Study 1 and are displayed in Table 4. As in Study 1, the most complex DynAffect model had by far the best fit and was used in further analyses.

How well does the selected model fit the observed data? Comparing observed and replicated core affect trajectories. Analogous to Study 1, Figure 5 displays the observed and two replicated core affect trajectories for 10 participants that are characterized by varying values in the parameters of the DynAffect model. As can be seen from this figure, the overall shapes of the trajectories show strong correspondence. The correlation between the observed and replicated (based on 1,000 simulated data sets) visit frequencies in the $3 \times 3$ sectors of the core affect space averaged 0.84 across participants $(S D=0.14$, average $r=.51$ for a $9 \times 9$ grid), again demonstrating that the DynAffect model was able to capture how often people are in qualitatively different feeling when sampled frequently across daily life.

Next, Figure 6 shows the vector field for the observed and (20) simulated data sets. As in Study 1, it can be observed that close to the population home base, the average speed is very small, but it increases when moving away from the home base. Again, the replications (grey lines) show the same properties and average as the observed vectors.

Predictions of future data points based on the selected model: Prediction of the final observation. We estimated the DynAffect model without the last observation and predicted from that model the last point for each person 1,000 times. For the pleasantness dimension, the observed last point fell into the $95 \%$ prediction interval in $82 \%$ of the cases and, for the activation, in $95 \%$ of the cases, indicating a good to very good prediction rate.

Descriptive statistics and correlations with trait measures. Table 5 displays the values of the DynAffect parameters at the population level and the intercorrelations between parameters.

Table 2

Population-Level Means of the Posterior Distributions (Point Estimate), Posterior Standard Deviations (Uncertainty), and Intercorrelations Between the Person-Specific DynAffect Parameters in Study $1(N=79)$

\begin{tabular}{|c|c|c|c|c|c|c|c|c|}
\hline Parameter & $M$ & $S D$ & 1 & 2 & 3 & 4 & 5 & 6 \\
\hline 1. Valence home base & 5.95 & 0.09 & - & & & & & \\
\hline 2. Arousal home base & 4.30 & 0.10 & $-.50^{* * * *}$ & - & & & & \\
\hline 3. Valence variability & 3.21 & 0.31 & -.17 & .08 & 一 & & & \\
\hline 4. Arousal variability & 4.28 & 0.30 & $.27^{* *}$ & -.18 & $.47^{* * * *}$ & - & & \\
\hline 5. Valence attractor strength & 0.026 & 0.003 & $.24^{*}$ & -.07 & -.05 & .02 & - & \\
\hline 6. Arousal attractor strength & 0.025 & 0.003 & -.03 & -.04 & .16 & .04 & .19 & - \\
\hline
\end{tabular}


Table 3

Correlations (With Corresponding p Values in Parentheses) Between DynAffect Parameters and the Dispositional Measures in Study $1(N=79)$

\begin{tabular}{|c|c|c|c|c|c|c|}
\hline $\begin{array}{l}\text { Dispositional } \\
\text { measure }\end{array}$ & $\begin{array}{c}\text { Valence } \\
\text { home base }\end{array}$ & $\begin{array}{c}\text { Arousal } \\
\text { home base }\end{array}$ & $\begin{array}{c}\text { Valence } \\
\text { variability }\end{array}$ & $\begin{array}{c}\text { Arousal } \\
\text { variability }\end{array}$ & $\begin{array}{l}\text { Valence attractor } \\
\text { strength }\end{array}$ & $\begin{array}{c}\text { Arousal attractor } \\
\text { strength }\end{array}$ \\
\hline Neuroticism & $-.386(.000)$ & $.230(.042)$ & $.278(.013)$ & $-.046(.688)$ & $-.098(.392)$ & $-.175(.122)$ \\
\hline Extraversion & $.288(.010)$ & $-.197(.083)$ & $-.003(.979)$ & $.156(.171)$ & $.034(.766)$ & $.085(.457)$ \\
\hline Positive affect & $.446(.000)$ & $-.125(.272)$ & $.025(.830)$ & $.097(.394)$ & $.064(.573)$ & $.117(.304)$ \\
\hline Negative affect & $-.227(.044)$ & $.104(.360)$ & $.283(.011)$ & $-.098(.388)$ & $.076(.503)$ & $.065(.567)$ \\
\hline Self-esteem & $.356(.001)$ & $-.262(.019)$ & $-.253(.024)$ & $.025(.826)$ & $.058(.614)$ & $.144(.207)$ \\
\hline Self-esteem variability & $.069(.548)$ & $-.105(.358)$ & $.393(.000)$ & $.242(.032)$ & $.158(.165)$ & $.096(.398)$ \\
\hline Satisfaction with life & $.247(.028)$ & $-.178(.116)$ & $-.185(.103)$ & $.061(.595)$ & $.066(.562)$ & $.058(.614)$ \\
\hline Reappraisal & $.034(.763)$ & $.014(.901)$ & $-.212(.061)$ & $-.120(.292)$ & $.160(.159)$ & $.310(.005)$ \\
\hline Suppression & $-.146(.199)$ & $.230(.008)$ & $.125(.273)$ & $-.035(.760)$ & $-.017(.879)$ & $-.034(.764)$ \\
\hline Rumination & $-.080(.482)$ & $-.042(.711)$ & $.068(.552)$ & $-.177(.118)$ & $-.011(.925)$ & $-.226(.045)$ \\
\hline
\end{tabular}

Note. Significant correlations $(p<.05)$ are set in bold.

Again, participants' home base was slightly positive and of medium arousal, and the participants displayed substantial amount of variability, with arousal variability again being larger than valence variability. Of specific interest, attractor strength values were larger compared to Study 1, suggesting that stronger attractor forces may be at work on shorter time-scale core affect changes. To illustrate, Figure 7 displays the $95 \%$ credibility intervals for valence and arousal attractor strength parameters in Studies 1 and 2. The figure clearly shows very limited overlap between the intervals, suggesting that attractor strength is indeed substantially larger when based on data collected over shorter time intervals. Valence variability and arousal variability were again positively correlated, as were valence and arousal attractor strength and arousal variability and valence home base. Unlike in Study 1, the negative correlation between valence and arousal home base was substantially lower and not significant in this sample. Despite the differences, the overall pattern of correlations between the DynAffect parameters was highly similar to that in Study 1 (the correlation between the intercorrelations reported in Studies 1 and 2 equals .68, $N=15$, $p<.001)$.

The correlations between the person-specific estimates and the dispositional questionnaires are reported in Table 6. With some exceptions, a pattern of correlations emerged that was similar to the one observed in Study 1. The valence of the home base was positively related to extraversion (albeit marginally significant), positive affect, self-esteem, and satisfaction with life and nega- tively to neuroticism and suppression (but not to negative affect, unlike in Study 1). The relationships between home-base arousal and neuroticism and between self-esteem and suppression were not replicated, however. Valence variability was again correlated with neuroticism and other indicators of maladjustment such as negative affect and low self-esteem. As in Study 1, arousal variability was not related to such indices (but did show a marginally significant positive correlation with life satisfaction). As in Study 1, arousal attractor strength was related to reappraisal but, unlike in Study 1, not to rumination. Taking into account the lower power and therefore higher significance threshold in Study 2, the overall correlation pattern nevertheless showed a strong overall correspondence to that of Study 1: The correlation between the correlations reported in Study 1 (leaving out those with self-esteem stability) and Study 2 equaled $.66(N=54, p<.0001)$.

\section{Discussion}

The findings from this study demonstrate that the DynAffect account also captures individual differences in affective changes and trajectories when these changes are considered on a much shorter time scale compared to Study 1. The fact that the same processes and patterns underlie core affect dynamics on different time scales may suggest that core affect may to some extent be self-similar across time scales (Guastello \& Liebovitch, 2009). An interesting difference between the findings from Studies 1 and 2 is

Table 4

Relative Goodness-of-Fit DIC Measure for the DynAffect Diffusion Model and for Five Alternative Less Complex Models (Study 2)

\begin{tabular}{|c|c|c|c|c|}
\hline Model & Home base & Variability & Attractor strength & Estimated DIC \\
\hline DynAffect & random & random & random & $-6,697$ \\
\hline Alternative 1 & random & random & fixed & $-5,128$ \\
\hline Alternative 2 & random & fixed & random & $-4,378$ \\
\hline Alternative 3 & random & fixed & fixed & 10,379 \\
\hline Alternative 4 & random & random & 0 & 7,906 \\
\hline Alternative 5 & random & random & infinite & 42,258 \\
\hline
\end{tabular}

Note. Random indicates that the parameters vary across persons, and fixed indicates that all persons posses the same value. The best fitting model is displayed in bold. DIC = deviance information criterion. 


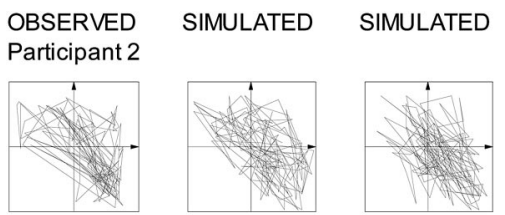

Participant 8
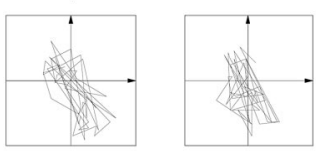

Participant 16
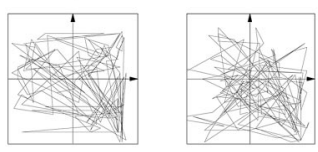

Participant 37
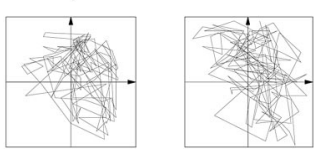

Participant 44
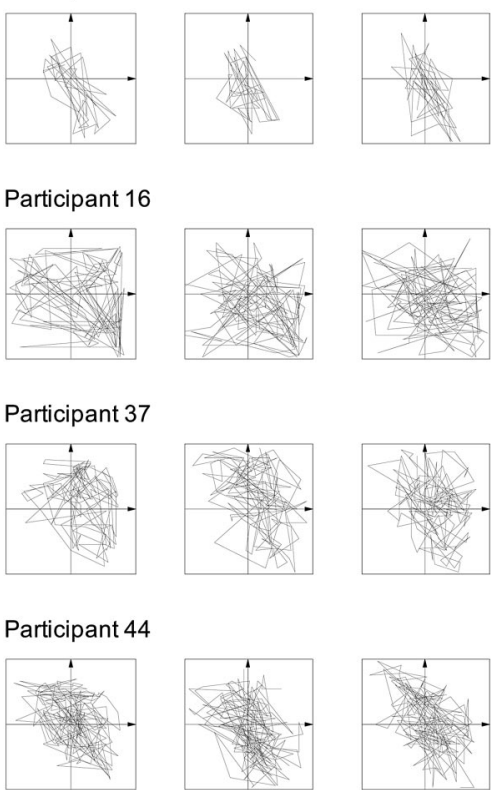

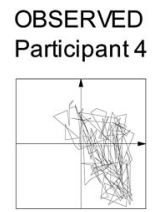

SIMULATED

SIMULATED
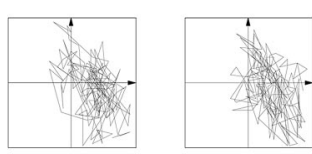

Participant 13
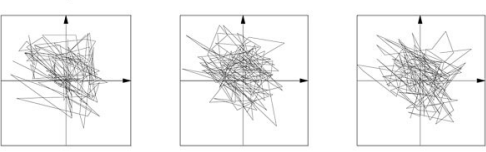

Participant 20
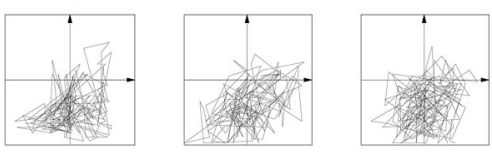

Participant 40
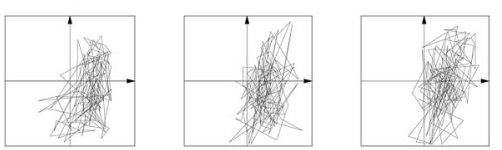

Participant 48
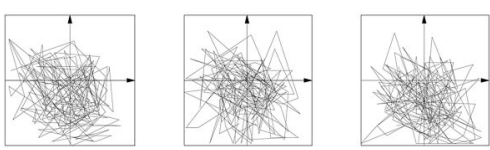

Figure 5. Observed core affect trajectories of 10 participants with two corresponding replicated trajectories based on data simulated under a DynAffect diffusion model implemented with the participants' estimated affective home base, variability, and attractor strength parameters (Study 2).

that the attractor strength seemed to be stronger for short-term affective changes compared to long-term changes. This interesting finding possibly marks the difference between the acute need for affect regulation during short-lived emotional episodes versus weaker attractor forces exerted on fluctuations following longer term changes.

Despite this difference, individual differences in affective home base, variability, and attractor strength showed interrelations and associations with personality and emotion traits highly comparable to those found in Study 1. This suggests that, at least in part, similar dispositional ways to feel, act, and regulate emotions may underlie these processes across shorter (minutes) and larger time scales (hours). However, there are several exceptions to this correspondence. Apart from the possibility that these exceptions signal a failure to replicate particular associations across the two studies, they may be indicative of differences between the processes underlying affect dynamics on different time scales. For instance, rumination was related to arousal attractor strength on a longer time scale but not on a shorter time scale. This may indicate that rumination particularly operates on longer term affective states and moods, supportive of its documented role in the etiology and continuation of mood disorders such as depression (e.g., Nolen-Hoeksema, 2000; Watkins, 2008). Second, neuroticism (as well as self-esteem) was not related to the arousal of participants' affective home base based on intensive sampling across 4 days but was positively related to home-base arousal based on 14 days of measurement. As previous research has shown that people's affect follows weekly cycles (Larsen \& Kasimatis, 1990), this may therefore indicate that neuroticism is related particularly to a stable component of arousal (obtained across 2 weeks of measurement) but not to a more volatile component independent of weekly cycles. Such an interpretation is supported by the consistent finding of a relationship between valence, but not arousal, variability and this personality trait across both studies. Finally, the habitual use of suppression was not found to be related to a higher arousal home base either. Together, arousal home-base levels seem to relate to dispositional measures especially when based on complete weeks of sampling, not less. This may suggest that arousal in particular may be strongly entrained to weekly cycles. Data based on less than a week of sampling may thus result in the confounding of individual differences with period of data collection during the week (reducing correlations with other dispositional individual differences). An important reminder for future research may therefore be that only arousal home base based on a week of sampling may validly capture tonic levels of people's experienced arousal.

\section{General Discussion}

We proposed DynAffect as a theoretical account of individual differences in the temporal dynamics of core affect. According to 


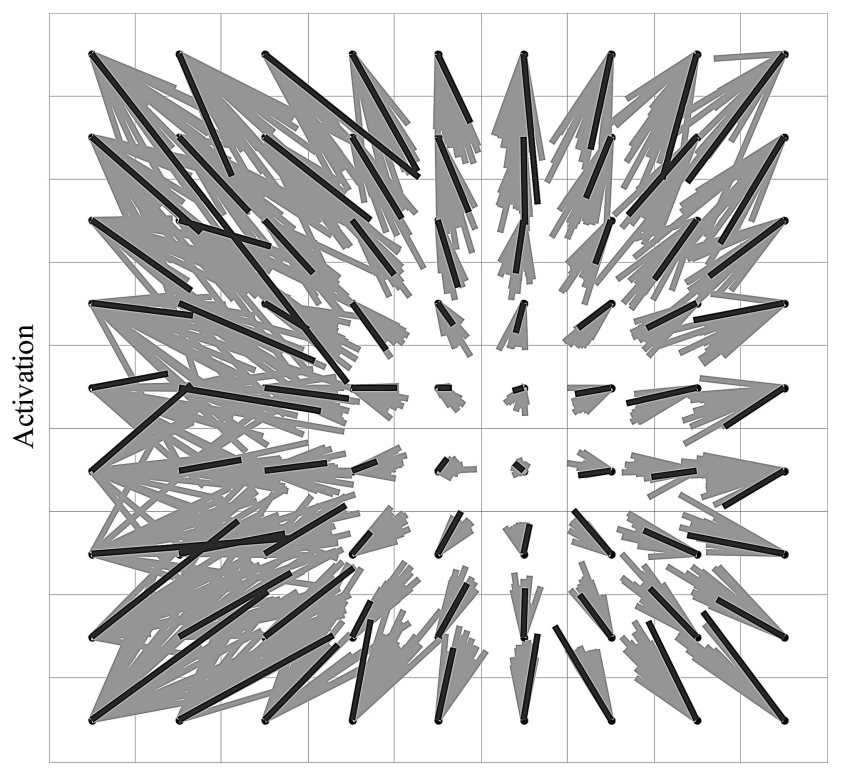

Pleasantness

Figure 6. Pooled vector field plot of the core affect space. The black lines reflect the observed vector (with the length reflecting the escape velocity and the direction the escape direction); the grey lines reflect vectors calculated on the basis of 20 simulated replications of the data under the DynAffect diffusion model (Study 2).

this account, the patterns and regularities with which people's feelings change across time are a function of people's affective home base, degree of affective variability, and attractor strength. Individual differences in these three key processes are responsible for observed individual differences in affect dynamics and trajectories and relate to personality, well-being, and habitual emotion regulation strategies in predictable ways. The DynAffect account was tested by applying a diffusion model approach to experiencesampling data reflecting core affect changes throughout participants' daily life over longer (Study 1) and shorter (Study 2) time scales. In both studies, the DynAffect model containing all parameters (home base, stochastic variability, and attractor strength, all varying across individuals) provided the best relative fit to the data; was capable of replicating the shape of individuals' core affect trajectories, how often they are in particular feeling states across time, and the dynamical forces that impinge on their feel- ings when in different feeling states; and was able to predict how they would feel next. The three relatively simple dynamical properties of affective home base, variability, and attractor strength put forward in our DynAffect account therefore seem capable of explaining to a large extent individual differences in temporal patterns and trajectories observed in people's affective experiences. The results furthermore provided novel insights into the nature of these processes.

When people feel close to their home base, up- or downward regulation is minimal (as illustrated in Figures 4 and 6). This suggests that the home base reflects the desired or optimal affective state for the individual, despite the fact that this state may be more unpleasant or arousing for some than for others. The further people's feeling state moves away from the home base, however, the larger the attractor strength becomes to return to this baseline level, suggesting indeed that the function of the home base lies in acting as an attractor or reference point for the regulation of affect. This generates interesting predictions that have been confirmed in experimental research. For instance, individuals with a more unpleasant home base should be less motivated or able to repair a negative affective state compared to individuals with a more pleasant home base and vice versa. Research by Hemenover (2003) indeed showed that neurotic and introverted individuals were characterized by slower repair (e.g., lower attractor strength) from elicited negative states, whereas low-neuroticism and extraverted individuals showed slower repair from elicited positive states.

Regarding affective variability, high affective variability is generally seen as an indicator of poorer well-being or adjustment (Kuppens et al., 2007). The present results show that this holds for valence variability (which was consistently found to be related to neuroticism, negative affectivity, and low self-esteem) but not necessarily for arousal variability. Indeed, despite being slightly larger in both studies, arousal variability was much less related to such variables (for similar results, see Kuppens et al., 2007) and was even found to be positively related to a pleasant home base in both studies (as well as marginally to life satisfaction in Study 2). These findings therefore provide the first evidence of an instance in which affective instability (i.e., in terms of arousal) has adaptive correlates. Experiencing large fluctuations in arousal clearly seems less harmful compared to experiencing fluctuations in pleasure. This may suggest that fluctuations between engagement and disengagement of goal pursuit (possibly reflected by different arousal values; e.g., Kuppens, 2008) may be less detrimental or even

Table 5

Population-Level Means of the Posterior Distributions (Point Estimate), Posterior Standard Deviations (Uncertainty), and Intercorrelations Between the Person-Specific DynAffect Parameters in Study $2(N=60)$

\begin{tabular}{lllllllll}
\hline \multicolumn{1}{c}{ Parameter } & $M$ & $S D$ & 1 & 2 & 3 & 4 & 5 & 6 \\
\hline 1. Valence home base & 5.82 & 0.08 & - & & & & \\
2. Arousal home base & 4.23 & 0.10 & -.24 & - & & & \\
3. Valence variability & 2.67 & 0.24 & -.20 & -.15 & - & & \\
4. Arousal variability & 4.29 & 0.30 & $.30^{*}$ & -.15 & $.29^{*}$ & - & \\
5. Valence attractor strength & 0.043 & 0.008 & -.07 & -.25 & $.27^{*}$ & .15 & - & \\
6. Arousal attractor strength & 0.049 & 0.012 & -.11 & $-.34^{*}$ & $.43^{* * *}$ & .01 & $.43^{* *}$ & - \\
${ }^{*} p<.05 .{ }^{* * *} p<.01$. & & & & & & &
\end{tabular}


a

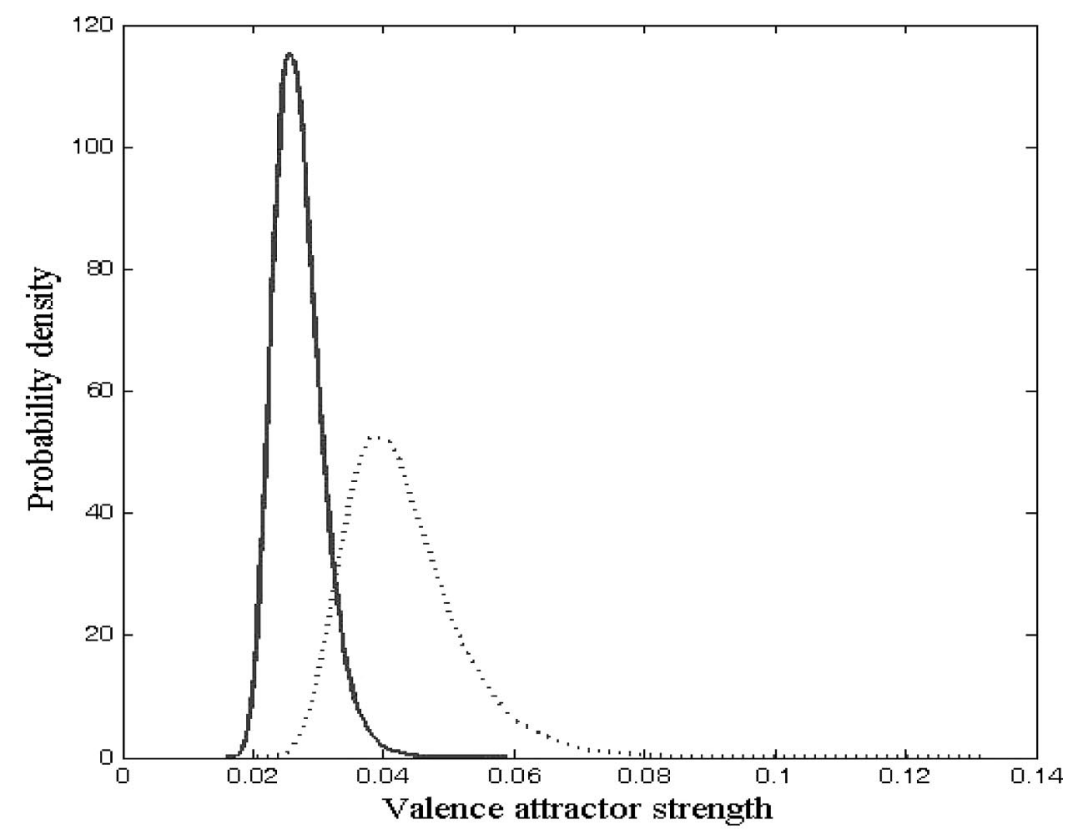

b

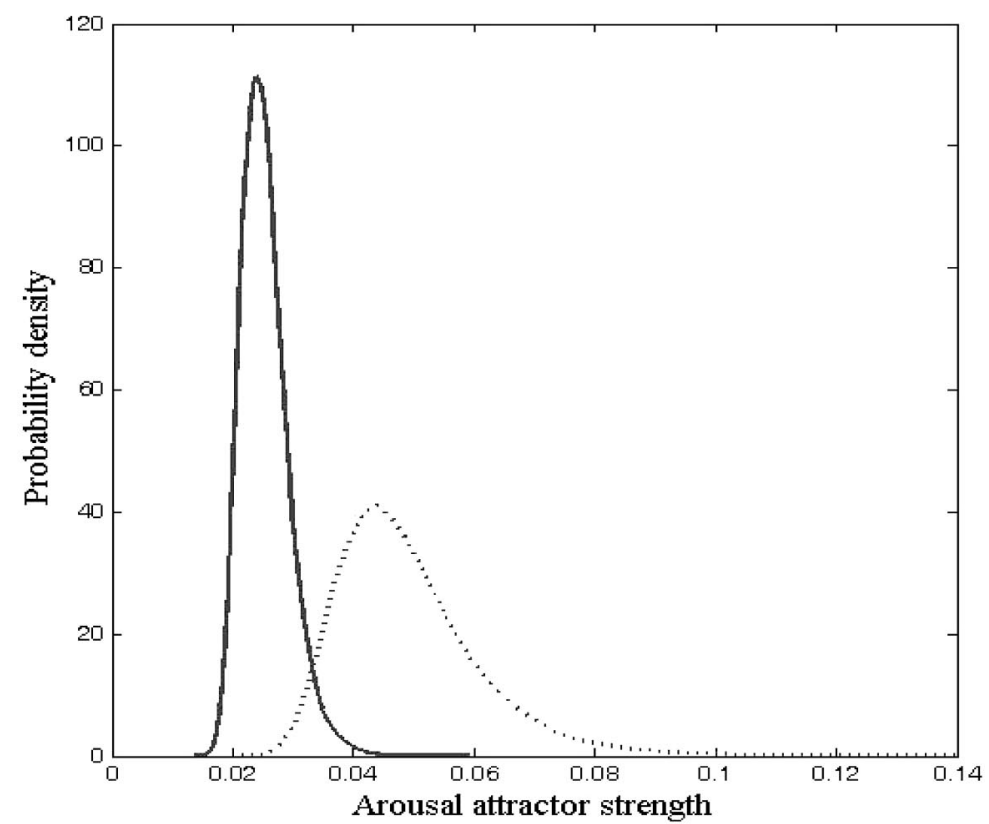

Figure 7. 95\% credibility intervals for the parameters valence attractor strength (a) and arousal attractor strength (b) in Study 1 (solid line) and Study 2 (dotted line) based on smoothed posterior densities.

adaptive to psychological well-being compared to changes in goal threats and opportunities that are signaled by changes in valence. Also, arousal variability may signal increased emotional intelligence (in the sense of the ability to distinguish between different like-valenced states based on their arousal level; see, e.g., the concept of arousal focus; e.g., Barrett, Quigley, Bliss-Moreau, \&
Aronson, 2004). In line with the previous, this may signal that changes in arousal are less maladaptive and therefore need to be regulated less compared to changes in valence.

The finding that the principle of an attractor is crucial for the understanding of core affect dynamics supports the idea that affect is not free floating but intrinsically regulated, at least in terms of 
Table 6

Correlations (With Corresponding p Values in Parentheses) Between DynAffect Parameters and the Dispositional Measures in Study $2(N=60)$

\begin{tabular}{|c|c|c|c|c|c|c|}
\hline $\begin{array}{l}\text { Dispositional } \\
\text { measure }\end{array}$ & $\begin{array}{c}\text { Valence } \\
\text { home base }\end{array}$ & $\begin{array}{c}\text { Arousal } \\
\text { home base }\end{array}$ & $\begin{array}{c}\text { Valence } \\
\text { variability }\end{array}$ & $\begin{array}{c}\text { Arousal } \\
\text { variability }\end{array}$ & $\begin{array}{l}\text { Valence attractor } \\
\text { strength }\end{array}$ & $\begin{array}{c}\text { Arousal attractor } \\
\text { strength }\end{array}$ \\
\hline Neuroticism & $-.413(.001)$ & $.096(.465)$ & $.261(.044)$ & $-.063(.633)$ & $-.007(.961)$ & $.067(.612)$ \\
\hline Extraversion & $.251(.053)$ & $-.087(.509)$ & $.105(.426)$ & $.120(.361)$ & $.008(.950)$ & $.204(.119)$ \\
\hline Positive affect & $.303(.019)$ & $.079(.550)$ & $.028(.829)$ & $.194(.139)$ & $.107(.417)$ & $.050(.702)$ \\
\hline Negative affect & $-.136(.302)$ & $.146(.265)$ & $.309(.016)$ & $.109(.406)$ & $.091(.491)$ & $.057(.667)$ \\
\hline Self-esteem & $.257(.047)$ & $.070(.594)$ & $-.235(.071)$ & $.141(.283)$ & $.125(.343)$ & $-.198(.129)$ \\
\hline Satisfaction with life & $.488(.000)$ & $-.099(.450)$ & $.010(.938)$ & $.247(.057)$ & $.073(.579)$ & $-.083(.526)$ \\
\hline Reappraisal & $.211(.105)$ & $-.138(.292)$ & $.050(.703)$ & $.168(.199)$ & $.076(.565)$ & $.268(.039)$ \\
\hline Suppression & $-.276(.033)$ & $.052(.695)$ & $.012(.928)$ & $-.103(.432)$ & $.030(.821)$ & $.184(.159)$ \\
\hline Rumination & $-.181(.165)$ & $.037(.779)$ & $.087(.510)$ & $-.086(.515)$ & $-.028(.835)$ & $.083(.528)$ \\
\hline
\end{tabular}

Note. Significant correlations $(p<.05)$ are set in bold.

possessing an attractor dynamic, the propensity to return to a baseline level. The presence of such a principle for regulating affect dynamics can be considered to be highly functional and adaptive, given the obvious disrupting consequences of extreme affective states that would disrupt normal functioning or of affect to linger endlessly without a set-point state to return to. Moreover, attractor regulation may have a hedonic component to it itself, in the sense that returning to baseline or a reference affective state is usually experienced as pleasant and experiences that move away from the reference point as unpleasant (Carver \& Scheier, 1990; Panksepp, 1998). One of the most striking findings with respect to individual differences in attractor strength is that the emotion regulation strategy of reappraisal was consistently found to be related to increased arousal attractor strength but not particularly to valence attractor strength. In other words, reappraisal efforts seem to impinge mainly on the arousal properties of affective experiences and less on hedonistic properties. This finding is ironic, given that valence variability in particular was found to be related to lower well-being. The aspect of core affect that reappraisal seems to target most effectively, therefore, may not be the one that has most beneficial effects. A crucial task for future research will be to identify emotion regulation styles that do affect valence attractor strength, given its obvious function of maintaining hedonic balance.

\section{Relation to Psychopathology}

Besides describing normal variation in emotion dynamics, the DynAffect account can provide a useful heuristic for characterizing the emotional phenomenology of psychopathological disorders that are, at their core, characterized by affective dysfunctioning. Indeed, the different expression forms of affective dysfunctioning evident in such disorders can be considered to reflect distinct combinations of (extreme values taken on) the dynamical properties of affective home base, variability, and attractor strength. In particular, major depression involves prolonged negative mood accompanied by a lack of activity and a loss of interest and enthusiasm (e.g., American Psychiatric Association, 1994). Moreover, recent research on the emotion dynamics in depression provided evidence that this disorder is characterized by increased levels of emotional inertia (Kuppens et al., 2010). In other words, the emotional phenomenology of depression involves a negatively valenced, low-arousal affective home base in combination with low attractor strength. Anxiety disorders, in turn, are characterized by chronic anxiety, exaggerated worry, and tension (American Psychiatric Association, 1994) and therefore primarily seem to involve a negatively valenced but highly aroused affective home base. The core pathology underlying borderline personality disorder is considered to be affective instability, in combination with intense negative mood (American Psychiatric Association, 1994; Nica \& Links, 2009; Trull et al., 2008). Borderline personality disorder, therefore, can be thought to primarily involve extreme levels of affect variability in combination with a negatively valenced home base. Bipolar mood disorder, finally, can be expected to involve more than one home base or attractor between which the individual switches as a function of phases of depression (extremely negatively valenced, low-arousal home base) and mania (extremely positively valenced, high-arousal home base; e.g., American Psychiatric Association, 1994; Hamaker, Grasman, \& Kamphuis, 2010). As such, the DynAffect account may provide a unified framework not only to account for normal variation in affect dynamics, but also to identify and classify the different maladaptive forms of affect dynamics implicated in these disorders in terms of their affective home base, variability, and attractor strength. Future research with such populations is needed to examine how the different dynamic processes indeed map onto such disorders.

\section{Limitations and Future Directions}

The proposed DynAffect account is intended to comprehensively capture the intricacies and variable expressions of individual differences in affect dynamics on the one hand while relying on a relatively parsimonious explanatory model on the other. As a result of this, several processes may not be incorporated that may nevertheless be important for the understanding of affect dynamics. For one, affect dynamics are evidently strongly driven by concurrent external and internal events. Although the DynAffect model takes the summation of these influences into account, it may be desirable to explicitly incorporate time-varying factors to enable the study of how momentary processes such as appraisals and ongoing emotion regulation efforts impact the unfolding of affect over time. Second, it can be argued that the symmetry that characterizes attractor strength may not be the most realistic. That is, it 
is not unlikely that the processes underlying, for instance, the attractor pull of negative feelings to a more positive home base (reflecting changes from more to less unpleasant affect) are not the same as those underlying the downward attractor pull exerted on very positive emotions (reflecting changes from more to less pleasant affect). Third, our account rests on the assumption that people's affect dynamics are characterized by a single-point attractor that is stable across time. Yet it is possible that a person's affect space may actually be characterized by multiple attractor basins of varying strength or that the attractor characteristics (location, strength) can vary across time as a function of tippingpoint shifts in the system (changing its entire operating characteristics). At present, such finer distinctions have not been taken into account as they would have added additional complexity to the account and modeling approach. Work is currently underway, however, that will allow the modeling of concurrent factors, asymmetric attractor strength, and multiple, regime-switching attractors that we hope will allow an even more fine-grained examination of the processes driving affect dynamics. Comparison between such models, as well as with yet other, alternative dynamical systems models (e.g., fractal Brownian motion, power-law distribution) can significantly help to uncover the patterns underlying affect dynamics. Finally, more research is needed to examine how the nature of the DynAffect processes may vary as a function of the time scale (and sampling scheme) of the observed affect dynamics.

\section{Conclusion}

Research is starting to unravel the dynamical regularities and processes that drive the changes and fluctuations in how people feel. The importance of understanding these processes lies in the insights it can deliver in the functions of affect and how people regulate their feelings as well as insight into the characteristics of maladaptive emotional functioning such as are observed in mood disorders. With the present article, we have proposed a theoretical account that identifies the key processes that underlie individual differences in affect dynamics, labeled DynAffect, and have reported findings in support of this account and about how the hypothesized processes are positioned in relation to broader personality and emotion dispositions when considered across both longer and shorter time scales. The key DynAffect processesaffective home base, affective variability, and attractor strengthreflect relatively simple principles yet have proved to be successful in capturing the wide variety of patterns and regularities that characterize people's affective fluctuations in normal daily life. This approach has also led to a number of novel insights into the dynamics of affect, such as the crucial role of an attractor state for providing coherence in the affective system, the potential adaptive nature of arousal variability, and the fact that reappraisal seems to mainly impinge on arousal rather than valence. In sum, the DynAffect account may provide an overarching framework for interpreting previous findings and inspire future studies on the dynamics of affect by identifying the major processes underlying individual differences in affect dynamics.

\section{References}

American Psychiatric Association. (1994). Diagnostic and statistical manual of mental disorders (4th ed.). Washington, DC: Author.
Barndorff-Nielsen, O. E., \& Shepard, N. (2001). Non-Gaussian OrnsteinUhlenbeck models and some of their uses in financial economics (with discussion). Journal of the Royal Statistical Society, Series B, 63, 167241. doi:10.1111/1467-9868.00282

Barrett, L. F., \& Barrett, D. J. (2001). Computerized experience-sampling: How technology facilitates the study of conscious experience. Social Science Computer Review, 19, 175-185.

Barrett, L. F., \& Bliss-Moreau, E. (2009). Affect as a psychological primitive. Advances in Experimental Social Psychology, 41, 167-218. doi:10.1016/S0065-2601(08)00404-8

Barrett, L. F., Mesquita, B., Ochsner, K. N., \& Gross, J. J. (2007). The experience of emotion. Annual Review of Psychology, 58, 373-403. doi:10.1146/annurev.psych.58.110405.085709

Barrett, L. F., Quigley, K. S., Bliss-Moreau, E., \& Aronson, K. R. (2004). Interoceptive sensitivity and self-reports of emotional experience. Journal of Personality and Social Psychology, 87, 684-697. doi:10.1037/ 0022-3514.87.5.684

Barrett, L. F., \& Russell, J. A. (1999). The structure of current affect: Controversies and emerging consensus. Current Directions in Psychological Science, 8, 10-14. doi:10.1111/j.0963-7214.2006.00411.x

Blackwell, P. G. (1997). Random diffusion models for animal movements. Ecological Modelling, 100, 87-102. doi:10.1016/S0304-3800(97)00153-1

Boker, S. M., \& Wenger, M. J. (2007). Data analytic techniques for dynamical systems. Mahwah, NJ: Erlbaum.

Bolger, N., Davis, A., \& Rafaeli, E. (2003). Diary methods: Capturing life as it is lived. Annual Review of Psychology, 54, 579-616. doi:10.1146/ annurev.psych.54.101601.145030

Brown, R. (1828). A brief account of microscopical observations made in the months of June, July and August, 1827, on the particles contained in the pollen of plants; and on the general existence of active molecules in organic and inorganic bodies. Philosophical Magazine, 4, 161-173.

Butler, E. A., Egloff, B., Wilhelm, F. H., Smith, N. C., Erickson, E. A., \& Gross, J. J. (2003). The social consequences of expressive suppression. Emotion, 3, 48-67. doi:10.1037/1528-3542.3.1.48

Cacioppo, J. T., \& Gardner, W. L. (1999). Emotion. Annual Review of Psychology, 50, 191-214. doi:10.1146/annurev.psych.50.1.191

Carver, C. S., \& Scheier, M. F. (1990). Origins and functions of positive and negative affect: A control-process view. Psychological Review, 97, 19-35. doi:10.1037/0033-295X.97.1.19

Chow, S.-M., Ram, N., Boker, S. M., Fujita, F., \& Clore, G. (2005). Emotion as a thermostat: Representing emotion regulation using a damped oscillator model. Emotion, 5, 208-225. doi:10.1037/15283542.5.2.208

Cohen, S., \& Pressman, S. D. (2006). Positive affect and health. Current Directions in Psychological Science, 15, 122-125. doi:10.1111/j.09637214.2006.00420.x

Cox, D. R., \& Miller, H. D. (1965). The theory of stochastic processes. London, England: Chapman \& Hall.

Csikszentmihalyi, M., \& Larsen, R. (1987). Validity and reliability of the experience sampling method. Journal of Nervous and Mental Disease, 175, 526-536. doi:10.1097/00005053-198709000-00004

Davidson, R. J. (1998). Affective style and affective disorders: Perspectives from affective neuroscience. Cognition \& Emotion, 12, 307-330. doi:10.1080/026999398379628

Davidson, R. J. (2003). Darwin and the neural bases of emotion and affective style. Annals of the New York Academy of Sciences, 1000, 316-336. doi:10.1196/annals.1280.014

Davidson, R. J., Jackson, D. C., \& Kalin, N. H. (2000). Emotion, plasticity, context, and regulation: Perspectives from affective neuroscience. Psychological Bulletin, 126, 890-909. doi:10.1037/0033-2909.126.6.890

Davidson, R. J., Pizzagalli, D., Nitschke, J. B., \& Putnam, K. (2002). Depression: Perspectives from affective neuroscience. Annual Review of Psychology, 53, 545-574. doi:10.1146/annurev.psych.53.100901.135148

Davidson, R. J., Putnam, K. M., \& Larson, C. L. (2000, July 28). Dys- 
function in the neural circuitry of emotion regulation: A possible prelude to violence. Science, 289, 591-594. doi:10.1126/science.289.5479.591

Denissen, J. J. A., Butalid, L., Penke, L., \& van Aken, M. A. G. (2008). The effects of weather on daily mood: A multilevel approach. Emotion, 8, 662-667. doi:10.1037/a0013497

Diener, E., \& Diener, C. (1996). Most people are happy. Psychological Science, 7, 181-185. doi:10.1111/j.1467-9280.1996.tb00354.x

Diener, E., Emmons, R. A., Larsen, R. J., \& Griffin, S. (1985). The Satisfaction With Life Scale. Journal of Personality Assessment, 49, 71-75. doi:10.1207/s15327752jpa4901_13

Diener, E., Oishi, S., \& Lucas, R. E. (2003). Personality, culture, and subjective well-being: Emotional and cognitive evaluations of life. Annual Review of Psychology, 54, 403-425. doi:10.1146/annurev .psych.54.101601.145056

Diener, E., Smith, H., \& Fujita, F. (1995). The personality structure of affect. Journal of Personality and Social Psychology, 69, 130-141. doi:10.1037/0022-3514.69.1.130

Eaton, L. G., \& Funder, D. C. (2001). Emotional experience in daily life: Valence, variability, and rate of change. Emotion, 1, 413-421. doi: 10.1037/1528-3542.1.4.413

Eid, M., \& Diener, E. (1999). Intraindividual variability in affect: Reliability, validity, and personality correlates. Journal of Personality and Social Psychology, 76, 662-676. doi:10.1037/0022-3514.76.4.662

Einstein, A. (1905). Über die von der molekularkinetischen Theorie der Wärme geforderte Bewegung von in ruhenden Flüssigkeiten suspendierten Teilchen [On the movement of a particle in resting fluid required by the molecular-kinetic theory of heat]. Annalen der Physik, 322, 549-560. doi:10.1002/andp.19053220806

Ekkekakis, P. (2009). The dual-mode theory of affective responses to exercise in metatheoretical context: I. Initial impetus, basic postulates, and philosophical framework. International Review of Sport and Exercise Psychology, 2, 73-94. doi:10.1080/17509840802705920

Fleeson, W. (2001). Toward a structure- and process-integrated view of personality: Traits as density distributions of states. Journal of Personality and Social Psychology, 80, 1011-1027. doi:10.1037/00223514.80.6.1011

Fleeson, W. (2004). Moving personality beyond the person-situation debate: The challenge and the opportunity of within-person variability. Current Directions in Psychological Science, 13, 83-87. doi:10.1111/ j.0963-7214.2004.00280.x

Fredrickson, B. L., \& Losada, M. F. (2005). Positive affect and the complex dynamics of human flourishing. American Psychologist, 60, 678-686. doi:10.1037/0003-066X.60.7.678

Frijda, N. H. (1986). The emotions. Cambridge, England: Cambridge University Press.

Frijda, N. H. (2007). The laws of emotion. Mahwah, NJ: Erlbaum.

Gardiner, C. W. (2004). Handbook of stochastic methods. New York, NY: Springer.

Gelman, A., Carlin, J., Stern, H., \& Rubin, D. (2004). Bayesian data analysis. New York, NY: Chapman \& Hall.

Gelman, A., \& Hill, J. (2007). Data analysis using regression and multilevel/hierarchical models. New York, NY: Cambridge University Press.

Grewe, O., Nagel, F., Kopiez, R., \& Altenmüller, E. (2007). Emotions over time: Synchronicity and development of subjective, physiological, and facial affective reactions to music. Emotion, 7, 774-788. doi:10.1037/ 1528-3542.7.4.774

Gross, J. J. (1998). Antecedent- and response-focused emotion regulation: Divergent consequences for experience, expression, and physiology. Journal of Personality and Social Psychology, 74, 224-237. doi: 10.1037/0022-3514.74.1.224

Gross, J. J. (2001). Emotion regulation in adulthood: Timing is everything. Current Directions in Psychological Science, 10, 214-219. doi:10.1111/ $1467-8721.00152$

Gross, J. J. (2002). Emotion regulation: Affective, cognitive, and social consequences. Psychophysiology, 39, 281-291. doi:10.1017/ S0048577201393198

Gross, J. J., \& John, O. P. (2003). Individual differences in two emotion regulation processes: Implications for affect, relationships, and wellbeing. Journal of Personality and Social Psychology, 85, 348-362. doi:10.1037/0022-3514.85.2.348

Gross, J. J., \& Levenson, R. W. (1997). Hiding feelings: The acute effects of inhibiting negative and positive emotion. Journal of Abnormal Psychology, 106, 95-103. doi:10.1037/0021-843X.106.1.95

Gross, J. J., \& Muñoz, R. F. (1995). Emotion regulation and mental health. Clinical Psychology: Science and Practice, 2, 151-164. doi:10.1111/ j.1468-2850.1995.tb00036.x

Guastello, S. J., Koopmans, M., \& Pincus, D. (Eds.). (2009). Chaos and complexity in psychology: The theory of nonlinear dynamical systems. New York, NY: Cambridge University Press.

Guastello, S. J., \& Liebovitch, L. S. (2009). Introduction to nonlinear dynamics and complexity. In S. J. Guastello, M. Koopmans, \& D. Pincus (Eds.), Chaos and complexity in psychology: The theory of nonlinear dynamical systems (pp. 1-40). New York, NY: Cambridge University Press.

Hamaker, E. L., Grasman, R. P. P. P., \& Kamphuis, J. H. (2010). Regimeswitching models to study psychological processes. In P. C. M. Molenaar \& K. M. Newell (Eds.), Individual pathways of change: Statistical models for analyzing learning and development (pp. 155168). Washington, DC: American Psychological Association.

Headey, B., \& Wearing, A. (1989). Personality, life events, and subjective well-being: Toward a dynamic equilibrium model. Journal of Personality and Social Psychology, 57, 731-739. doi:10.1037/00223514.57.4.731

Hemenover, S. H. (2003). Individual differences in rate of affect change: Studies in affective chronometry. Journal of Personality and Social Psychology, 85, 121-131. doi:10.1037/0022-3514.85.1.121

Hemenover, S. H., Augustine, A. A., Shulman, T., Tran, T. Q., \& Barlett, C. P. (2008). Individual differences in negative affect repair. Emotion, 8, 468-478. doi:10.1037/1528-3542.8.4.468

Hepburn, L., \& Eysenck, M. W. (1989). Personality, average mood and mood variability. Personality and Individual Differences, 10, 975-983. doi:10.1016/0191-8869(89)90062-7

Hoeksma, J. B., Oosterlaan, J., Schipper, E., \& Koot, H. (2007). Finding the attractor of anger: Bridging the gap between concepts and empirical data. Emotion, 7, 638-648. doi:10.1037/1528-3542.7.3.638

Hoekstra, H. A., Ormel, J., \& De Fruyt, F. (1996). NEO PI-R, NEO FFI Big Five Persoonlijkheidsvragenlijsten: Handleiding. [NEO-PI-R, NEOFFI personality questionnaires: Manual] Lisse, the Netherlands: Swets \& Zeitlinger.

Ickes, W., Snyder, M., \& Garcia, S. (1997). Personality influences on the choice of situations. In R. Hogan, J. A. Johnson, \& S. Briggs (Eds.), Handbook of personality psychology (pp. 165-195). San Diego, CA: Academic Press. doi:10.1016/B978-012134645-4/50008-1

Jones, C. J. (2006). P-technique factor analysis as a tool for exploring psychological health. In A. D. Ong \& M. H. M. Van Dulmen (Eds.), Oxford handbook of methods in positive psychology (pp. 3-11). New York, NY: Oxford University Press.

Kahneman, D., Krueger, A. B., Schkade, D. A., Schwarz, N., \& Stone, A. A. (2004, December 3). A survey method for characterizing daily life experience: The day reconstruction method. Science, 306, 1776-1780. doi:10.1126/science.1103572

Kernis, M. H. (2003). Toward a conceptualization of optimal self-esteem. Psychological Inquiry, 14, 1-26. doi:10.1207/S15327965PLI1401_01

Kernis, M. H., Cornell, D. P., Sun, C., Berry, A., \& Harlow, T. (1993). There's more to self-esteem than whether it is high or low: The importance of stability of self-esteem. Journal of Personality and Social Psychology, 65, 1190-1204. doi:10.1037/0022-3514.65.6.1190 
Koole, S. L. (2009). The psychology of emotion regulation: An integrative review. Cognition \& Emotion, 23, 4-41. doi:10.1080/02699930802619031

Kuppens, P. (2008). Individual differences in the relationship between pleasure and arousal. Journal of Research in Personality, 42, 10531059. doi:10.1016/j.jrp.2007.10.007

Kuppens, P., Allen, N. B., \& Sheeber, L. (2010). Emotional inertia and psychological maladjustment. Psychological Science, 21, 984-991.

Kuppens, P., Stouten, J., \& Mesquita, B. (2009). Individual differences in emotion components and dynamics: Introduction to the special issue. Cognition \& Emotion, 23, 1249-1258. doi:10.1080/02699930902985605

Kuppens, P., Tuerlinckx, F., Russell, J. A., \& Barrett, L. F. (2010). The relationship between valence and arousal in subjective experience. Manuscript submitted for publication.

Kuppens, P., Van Mechelen, I., Nezlek, J. B., Dossche, D., \& Timmermans, T. (2007). Individual differences in core affect variability and their relationship to personality and adjustment. Emotion, 7, 262-274. doi:10.1037/1528-3542.7.2.262

Larsen, R. J. (1987). The stability of mood variability: A spectral analytic approach to daily mood assessments. Journal of Personality and Social Psychology, 52, 1195-1204. doi:10.1037/0022-3514.52.6.1195

Larsen, R. J. (2000). Towards a science of mood regulation. Psychological Inquiry, 11, 129-141. doi:10.1207/S15327965PLI1103_01

Larsen, R. J., \& Diener, E. (1985). A multitrait-multimethod examination of affect structure: Hedonic level and emotional intensity. Personality and Individual Differences, 6, 631-636. doi:10.1016/01918869(85)90013-3

Larsen, R. J., \& Kasimatis, M. (1990). Individual differences in entrainment of mood to the weekly calendar. Journal of Personality and Social Psychology, 58, 164-171. doi:10.1037/0022-3514.58.1.164

Larsen, R. J., \& Ketelaar, T. (1991). Personality and susceptibility to positive and negative states. Journal of Personality and Social Psychology, 61, 132-140. doi:10.1037/0022-3514.61.1.132

Lee, M. D. (2008). Three case studies in the Bayesian analysis of cognitive models. Psychonomic Bulletin \& Review, 15, 1-15. doi:10.3758/ PBR.15.1.1

Lewis, M. D. (2005). Bridging emotion theory and neurobiology through dynamic modeling. Behavioral and Brain Sciences, 28, 169-194. doi: 10.1017/S0140525X0500004X

Lucas, R. E. (2007). Adaptation and the set point model of subjective well-being. Does happiness change after major life events? Current Directions in Psychological Science, 16, 75-79. doi:10.1111/j.14678721.2007.00479.x

Lykken, D. T., \& Tellegen, A. (1996). Happiness is a stochastic phenomenon. Psychological Science, 7, 186-189. doi:10.1111/j.14679280.1996.tb00355.x

Lyubomirsky, S., Sheldon, K. M., \& Schkade, D. (2005). Pursuing happiness: The architecture of sustainable change. Review of General Psychology, 9, 111-131. doi:10.1037/1089-2680.9.2.111

Mazzo, R. M. (2002). Brownian motion: Fluctuations, dynamics and applications. Oxford, England: Oxford University Press.

Mischel, W., \& Shoda, Y. (1995). A cognitive-affective system theory of personality: Reconceptualizing situations, dispositions, dynamics, and invariance in personality structure. Psychological Review, 102, 246268. doi:10.1037/0033-295X.102.2.246

Moskowitz, D. S., \& Zuroff, D. C. (2004). Flux, pulse, and spin: Dynamic additions to the personality lexicon. Journal of Personality and Social Psychology, 86, 880-893. doi:10.1037/0022-3514.86.6.880

Murray, G., Allen, N. B., \& Trinder, J. (2002). Longitudinal investigation of mood variability and the FFM: Neuroticism predicts variability in extended states of positive and negative affect. Personality and Individual Differences, 33, 1217-1228. doi:10.1016/S0191-8869(01)00217-3

Nesse, R. M., \& Berridge, K. C. (1997, October 3). Psychoactive drug use in evolutionary perspective. Science, 278, 63-66. doi:10.1126/ science. 278.5335 .63
Nesse, R. M., \& Ellsworth, P. C. (2009). Evolution, emotions, and emotional disorders. American Psychologist, 64, 129-139. doi:10.1037/ a0013503

Nezlek, J. B., \& Plesko, R. M. (2001). Day-to-day relationships among self-concept clarity, self-esteem, daily events, and mood. Personality and Social Psychology Bulletin, 27, 201-211. doi:10.1177/ 0146167201272006

Nica, E. I., \& Links, P. S. (2009). Affective instability in borderline personality disorder: Experience sampling findings. Current Psychiatry Reports, 11, 74-81. doi:10.1007/s11920-009-0012-2

Niedenthal, P. M. (2007, May 18). Embodying emotion. Science, 316, 1002-1005. doi:10.1126/science.1136930

Nolen-Hoeksema, S. (2000). The role of rumination in depressive disorder and mixed anxiety/depressive symptoms. Journal of Abnormal Psychology, 109, 504-511. doi:10.1037/0021-843X.109.3.504

Oosterwegel, A., Field, N., Hart, D., \& Anderson, K. (2001). The relation of self-esteem variability to emotion variability, mood, personality traits, and depressive tendencies. Journal of Personality, 69, 689-708. doi: $10.1111 / 1467-6494.695160$

Oravecz, Z., \& Tuerlinckx, F. (2008). Bayesian estimation of an OrnsteinUhlenbeck process based hierarchical model. In S. Balbi, G. Scepi, G. Russolillo, \& A. Stawinoga (Eds.), Proceedings of the Seventh International Conference on Social Science Methodology [CD-ROM]. Naples, Italy: University of Naples.

Oravecz, Z., \& Tuerlinckx, F. (in press). The linear mixed model and the hierarchical Ornstein-Uhlenbeck model: Some equivalences and differences. British Journal of Mathematical and Statistical Psychology.

Oravecz, Z., Tuerlinckx, F., \& Vandekerckhove, J. (2009). A hierarchical Ornstein-Uhlenbeck model for continuous repeated measurement data. Psychometrika, 74, 395-418. doi:10.1007/s11336-008-9106-8

Oravecz, Z., Tuerlinckx, F., \& Vandekerckhove, J. (in press). A hierarchical latent stochastic differential equation model for affective dynamics. Psychological Methods.

Oud, J. H. L. (2007). Comparison of four procedures to estimate the damped linear differential oscillator for panel data. In K. van Montfort, J. Oud, \& A. Satorra (Eds.), Longitudinal models in the behavioral and related sciences (pp. 19-39). Mahwah, NJ: Erlbaum.

Panksepp, J. (1998). Affective neuroscience: The foundations of human and animal emotions. New York, NY: Oxford University Press.

Pavot, W., \& Diener, E. (1993). Review of the Satisfaction With Life Scale. Psychological Assessment, 5, 164-172. doi:10.1037/10403590.5.2.164

Penner, L. A., Shiffman, S., Paty, J. A., \& Fritzsche, B. A. (1994). Individual differences in intraperson variability in mood. Journal of Personality and Social Psychology, 66, 712-721. doi:10.1037/00223514.66.4.712

Rodgers, J. L. (2010). The epistemology of mathematical and statistical modeling: A quiet methodological revolution. American Psychologist, 65, 1-12. doi:10.1037/a0018326

Rosenberg, M. (1989). Society and the adolescent self-image. Middletown, CT: Wesleyan University Press.

Rouder, J. N., Speckman, P. L., Sun, D., Morey, R. D., \& Iverson, G. (2009). Bayesian $t$-tests for accepting and rejecting the null hypothesis. Psychonomic Bulletin \& Review, 16, 225-237. doi:10.3758/ PBR.16.2.225

Rouder, J. N., Tuerlinckx, F., Speckman, P., Lu, J., \& Gomez, P. (2008). A hierarchical approach for fitting curves to response time measurements. Psychonomic Bulletin \& Review, 15, 1201-1208. doi:10.3758/ PBR.15.6.1201

Russell, J. A. (2003). Core affect and the psychological construction of emotion. Psychological Review, 110, 145-172. doi:10.1037/0033295X.110.1.145

Russell, J. A. (2009). Emotion, core affect, and psychological construction. Cognition \& Emotion, 23, 1259-1283. doi:10.1080/02699930902809375 
Russell, J. A., Weiss, A., \& Mendelsohn, G. A. (1989). Affect Grid: A single-item scale of pleasure and arousal. Journal of Personality and Social Psychology, 57, 493-502. doi:10.1037/0022-3514.57.3.493

Rusting, C. L., \& Larsen, R. J. (1995). Extraversion, neuroticism, and susceptibility to positive and negative affect. Personality and Individual Differences, 18, 321-329. doi:10.1016/0191-8869(94)00157-N

Rusting, C. L., \& Nolen-Hoeksema, S. (1998). Regulating responses to anger: Effects of rumination and distraction on angry mood. Journal of Personality and Social Psychology, 74, 790-803. doi:10.1037/00223514.74.3.790

Sapolsky, R. M. (2007). Stress, stress-related disease, and emotion regulation. In J. J. Gross (Ed.), Handbook of emotion regulation (pp. 606615). New York, NY: Guilford Press.

Scherer, K. R. (2000a). Emotion. In M. Hewstone \& W. Stroebe (Eds.), Introduction to social psychology: A European perspective (3rd ed., pp. 151-191). Oxford, England: Blackwell.

Scherer, K. R. (2000b). Emotions as episodes of subsystem synchronization driven by nonlinear appraisal processes. In M. D. Lewis \& I. Granic (Eds.), Emotion, development, and self-organization: Dynamic systems approaches to emotional development (pp. 70-99). New York, NY: Cambridge University Press. doi:10.1017/CBO9780511527883.005

Scherer, K. R. (2009). The dynamic architecture of emotion: Evidence for the component process model. Cognition \& Emotion, 23, 1307-1351. doi:10.1080/02699930902928969

Scherer, K. R., Dan, E. S., \& Flykt, A. (2006). What determines a feeling's position in affective space? A case for appraisal. Cognition \& Emotion, 20, 92-113. doi:10.1080/02699930500305016

Schroeder, M. (1991). Fractals, chaos, power laws: Minutes from an infinite paradise. New York, NY: Freeman.

Snijders, T. A. B., \& Bosker, R. J. (1999). Multilevel analysis: An introduction to basic and advanced multilevel modeling. London, England: Sage.

Spiegelhalter, D. J., Best, N. G., Carlin, B. P., \& van der Linde, A. (2002). Bayesian measures of model complexity and fit (with discussion). Journal of the Royal Statistical Society, Series B, 64, 583-639.

Spielberger, C. D., \& Sydeman, S. J. (1994). State-Trait Anxiety Inventory and State-Trait Anger Expression Inventory. In M. E. Maruish (Ed.), The use of psychological tests for treatment planning and outcome assessment (pp. 292-321). Hillsdale, NJ: Erlbaum.

Stone, A. A., Schwarz, J., Neale, J., Shiffman, S., Marco, C., Hickcox, M., .. . Cruise, L. (1998). A comparison of coping assessed by ecologic momentary assessment and retrospective recall. Journal of Personality and Social Psychology, 74, 1670-1680. doi:10.1037/00223514.74.6.1670

Strogatz, S. H. (1994). Nonlinear dynamics and chaos with applications to physics, biology, chemistry, and engineering. Cambridge, England: Westview.

Susman, E. J., Dorn, L. D., \& Chrousos, G. P. (1991). Negative affect and hormone levels in young adolescents: Concurrent and predictive perspectives. Journal of Youth and Adolescence, 20, 167-190. doi:10.1007/ BF01537607

Tamir, M. (2009). What do people want to feel and why? Current Directions in Psychological Science, 18, 101-105. doi:10.1111/j.14678721.2009.01617.x
Tanner, M. A. (1996). Tools for statistical inference: Methods for the exploration of posterior distributions and likelihood functions (3rd ed.). New York, NY: Springer.

Thagard, P., \& Nerb, J. (2002). Emotional gestalts: Appraisal, change, and the dynamics of affect. Personality and Social Psychology Review, 6, 274-282. doi:10.1207/S15327957PSPR0604_02

Trapnell, P. D., \& Campbell, J. D. (1999). Private self-consciousness and the five-factor model of personality: Distinguishing rumination from reflection. Journal of Personality and Social Psychology, 76, 284-304. doi:10.1037/0022-3514.76.2.284

Trull, T. J., Solhan, M. B., Tragesser, S. L., Jahng, S., Wood, P. K., Piasecki, T. M., \& Watson, D. (2008). Affective instability: Measuring a core feature of borderline personality disorder with ecological momentary assessment. Journal of Abnormal Psychology, 117, 647-661. doi: $10.1037 / \mathrm{a} 0012532$

Uhlenbeck, G. E., \& Ornstein, L. S. (1930). On the theory of Brownian motion. Physical Review, 36, 823-841. doi:10.1103/PhysRev.36.823

Vallacher, R. R., \& Nowak, A. (2009). The dynamics of human experience: Fundamentals of dynamical social psychology. In S. J. Guastello, M. Koopmans, \& D. Pincus (Eds.), Chaos and complexity in psychology: The theory of nonlinear dynamical systems (pp. 370-401). New York, NY: Cambridge University Press.

Vallacher, R. R., Read, S. J., \& Nowak, A. (2002). The dynamical perspective in personality and social psychology. Personality and Social Psychology Review, 6, 264-273. doi:10.1207/S15327957PSPR0604_01

van Montfort, K., Oud, J., \& Satorra, A. (2007). Longitudinal models in the behavioral and related sciences. New York, NY: Erlbaum.

Verduyn, P., Delvaux, E., Van Coillie, H., Tuerlinckx, F., \& Van Mechelen, I. (2009). Predicting the duration of emotional experience: Two experience sampling studies. Emotion, 9, 83-91. doi:10.1037/ a0014610

Verduyn, P., Van Mechelen, I., Tuerlinckx, F., Meers, K., \& Van Coillie, H. (2009). Intensity profiles of emotional experience over time. Cognition \& Emotion, 23, 1427-1443. doi:10.1080/02699930902949031

Watkins, E. R. (2008). Constructive and unconstructive repetitive thought. Psychological Bulletin, 134, 163-206. doi:10.1037/0033-2909.134.2.163

Watson, D. (2000). Mood and temperament. New York, NY: Guilford Press.

Watson, D., Clark, L. A., \& Tellegen, A. (1988). Development and validation of brief measures of positive and negative affect: The PANAS scale. Journal of Personality and Social Psychology, 54, 1063-1070. doi:10.1037/0022-3514.54.6.1063

Watson, D., \& Tellegen, A. (1985). Toward a consensual structure of mood. Psychological Bulletin, 98, 219-235. doi:10.1037/00332909.98.2.219

Witherington, D. C., \& Crichton, J. A. (2007). Frameworks for understanding emotions and their development: Functionalist and dynamic systems approaches. Emotion, 7, 628-637. doi:10.1037/1528-3542.7.3.628

Received December 17, 2009

Revision received May 19, 2010

Accepted May 24, 2010 